\title{
Real Number Graph Labellings with Distance Conditions *
}

\author{
Jerrold R. Griggs \\ Department of Mathematics \\ University of South Carolina \\ Columbia, SC 29208 USA \\ griggs@math.sc.edu
}

\author{
Teresa Xiaohua Jin \\ Department of Mathematics \\ University of South Carolina \\ Columbia, SC 29208 USA \\ jin2@math.sc.edu
}

Submitted: Dec 17, 2004; revised Oct. 8, 2005

AMS Subject Classifications: 05C78, 05C15, 90B18

\begin{abstract}
The theory of integer $\lambda$-labellings of a graph, introduced by Griggs and Yeh, seeks to model efficient channel assignments for a network of transmitters. To prevent interference, labels for nearby vertices must be separated by specified amounts $k_{i}$ depending on the distance $i, 1 \leq i \leq p$. Here we expand the model to allow real number labels and separations. The main finding (" $D$-set Theorem") is that for any graph, possibly infinite, with maximum degree at most $\Delta$, there is a labelling of minimum span in which all of the labels have the form $\sum_{i=1}^{p} a_{i} k_{i}$, where the $a_{i}$ 's are integers $\geq 0$. We show that the minimum span is a continuous function of the $k_{i}$ 's, and we conjecture that it is piecewise linear with finitely many pieces. Our stronger conjecture is that the coefficients $a_{i}$ can be bounded by a constant depending only on $\Delta$ and $p$. We offer results in strong support of the conjectures, and we give formulas for the minimum spans of several graphs with general conditions at distance two.
\end{abstract}

Keywords channel assignment, graph labelling, generalized coloring

\section{Integer Labellings with Distance Conditions}

A steadily growing body of literature has evolved in the past 15 years on efficient integer labellings of the vertices of a finite simple graph with restrictions not only on adjacent vertices-as is the case with traditional graph coloring-but also on vertices at distance two.

\footnotetext{
*Research supported in part by NSF grants DMS-0072187 and DMS-0302307. New address for second author: Department of Mathematics and Statistics, University of Vermont, Burlington, VT 05405 USA xiaohua.jin@uvm.edu . This research was also described in the second author's dissertation [19].
} 
In the traditional Channel Assignment Problem, introduced by Hale [17] and studied by Cozzens and Roberts [6] and many others, vertices of a graph $G=(V, E)$ correspond to transmitter locations, and their labels represent transmission channels. Adjacent vertices correspond to pairs of transmitters that interfere with each other due to their proximity. There is a given finite set $T$ of integers $\geq 0$, with $0 \in T$, of forbidden differences in channels for adjacent vertices. A vertex labelling $f: V \rightarrow \mathbb{Z}$ is a $T$-coloring provided that $|f(v)-f(w)| \notin T$ whenever vertices $v$ and $w$ are adjacent. Of course, one can select channels $f(v)$ that are very far apart, but this would require allocating a very large band of the frequency spectrum to the network. To optimize the assignment $f$ we seek to minimize the span

$$
\operatorname{sp}(f):=\max _{v \in V} f(v)-\min _{v \in V} f(v) .
$$

Note that labels need not be distinct. The set of labels used may contain gaps in the interval between the smallest and largest labels. It is the width of the interval, given by $\operatorname{sp}(f)$, that we seek to minimize.

In 1988 Roberts [27] described a new channel assignment problem, suggested by Tim Lanfear at NATO. This time we consider a given network of transmitters in the plane, with two different levels of interference. An integer channel is to be assigned to each transmitter such that channels for nearby transmitters (within, say, 100 miles) are distinct, and for very close transmitters (within, say, 50 miles) they differ by at least two. There is some spectral spreading of transmitters that decreases with distance between transmitters. Again, the goal is to construct a feasible labelling with minimum span.

For instance, Figure 1 shows a transmitter location in the plane with some other transmitters around it. The small circle is at 50 miles, while the large circle shows points at 100 miles from the center. A possible real-number channel assignment is shown, in which the central transmitter is assigned 0 , two other very close transmitters have labels at least two, and the two nearby transmitters that are not very close have labels at least one. This labelling satisfies the distance-labelling conditions for every pair of vertices, not just pairs involving the central transmitter. In the example, the label 0 is repeated, but at distance more than 100 miles from the center.

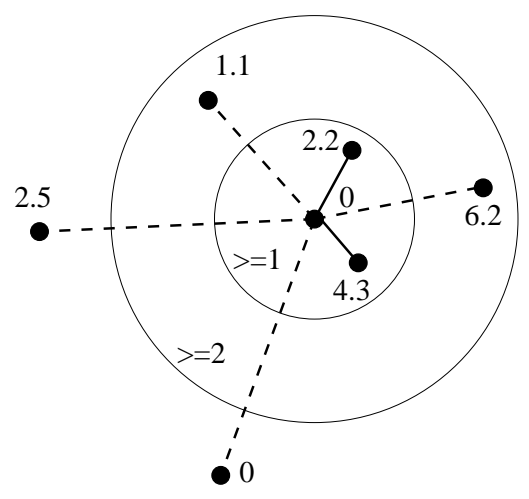

Figure 1: Labels for a planar transmitter network. 
Griggs [16] quickly discovered that this problem of labelling a planar transmitter network is quite challenging. In order to develop some heuristics for the real problem, he decided to investigate the natural graph analogue of the distance-labelling problem above, in which the vertices are the transmitters and adjacent vertices correspond to transmitters that are very close.

Specifically, for a finite simple graph $G=(V, E)$, consider a labelling $f: V \rightarrow \mathbb{Z}$ such that for all vertices $v, w \in V$,

$$
|f(v)-f(w)| \geq \begin{cases}2, & \text { if } \quad d(v, w)=1 \\ 1, & \text { if } d(v, w)=2,\end{cases}
$$

where $d(v, w)$ is the distance between $v$ and $w$ in $G$ (the minimum number of edges in any path from $v$ to $w$ ). For such labellings $f$, called $\lambda$-labellings of $G$, we seek to determine the minimum span, denoted $\lambda(G)$.

For instance, the 4 -cycle can be labelled by nonnegative integers by assigning 0 to one vertex and moving around the cycle, assigning each vertex the smallest label satisfying the conditions above. We end up using $0,2,4,6$ at consecutive vertices, but this "greedy firstfit" labelling is not optimal. We find that $\lambda\left(C_{4}\right)=4$ is achieved by labelling successive vertices by $0,4,1,3$.

By converting a planar network of transmitters-the problem of Roberts-to the graph problem, it is true that a pair of vertices at distance two in the graph corresponds to a pair of transmitters that are nearby, but not very close (at distance between 50 and 100 miles). However, a pair of transmitters in the plane can be close, but not very close, while their corresponding vertices in the graph are more than distance two apart. Their vertices need not even belong to the same component of the graph! For instance, in Figure 1 the transmitter labelled 1.1 is not very close to any other transmitter, so it would be isolated in the corresponding graph, although it is actually close to the transmitter at the center and to the one labelled 2.2.

Nonetheless, the study of $\lambda(G)$ for graphs $G$ should lead to good bounds and heuristics for efficiently labelling planar networks. Also, for some natural arrays the problems are equivalent. Especially, one particular array often used in practice for mobile communication networks assigns a hexagonal coverage region to each transmitter, with the hexagons fitting together in a honeycomb tiling. This is efficient in the sense of using a small number of transmitters. The graph corresponding to this example is called the triangular lattice, $\Gamma_{\triangle}$ (see Figure 2). In this case the graph problem does properly represent the real problem in the plane. Besides, the $\lambda$-labelling problem has turned out to be quite interesting on its own as a generalized graph coloring problem.

The natural generalization of $\lambda(G)$ to deal with multiple levels of interference was introduced by Griggs in the original paper with Yeh [16]: Let $\mathbb{N}$ denote the set of natural numbers, $\{0,1,2, \ldots\}$ (note that 0 is included). Given integers $k_{1}, \ldots, k_{p} \in \mathbb{N}$, let $L\left(k_{1}, \ldots, k_{p}\right)$ denote the set of labellings $f: V \rightarrow \mathbb{Z}$ such that for all $v, w \in V$

$$
|f(v)-f(w)| \geq k_{i}, \quad \text { if } d(v, w)=i \leq p .
$$

We may abbreviate this by $L(\mathbf{k})$, where $\mathbf{k}=\left(k_{1}, k_{2}, \ldots, k_{p}\right)$. Note that $L(\mathbf{k})$ always depends on the graph $G$ being considered. We seek the optimal span of any labelling $f$, 

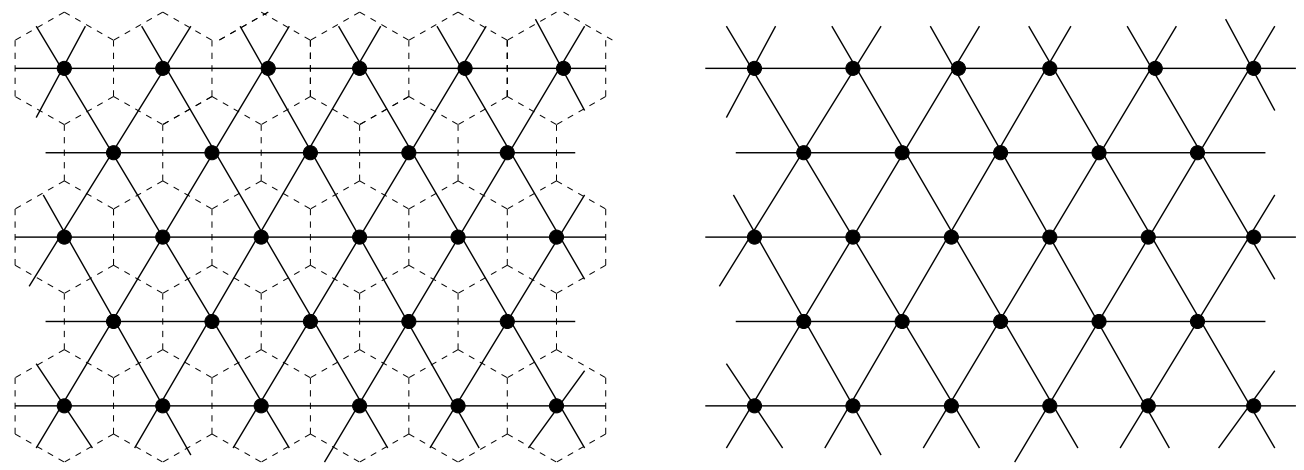

Figure 2: The honeycomb of hexagonal coverage regions of the plane (left); The corresponding triangular lattice $\Gamma_{\Delta}$ of transmitters (right).

denoted by

$$
\lambda\left(G ; k_{1}, \ldots, k_{p}\right):=\min _{f \in L(\mathbf{k})} \operatorname{sp}(f) .
$$

We also denote this lambda number of $G$ by $\lambda(G ; \mathbf{k})$. Alternate notation, also used by many authors, is $\lambda_{k_{1}, \ldots, k_{p}}(G)$. Another notation is in use for $p=2[8]$, where $\lambda_{k}^{j}(G)$ represents our $\lambda(G ; j, k)$. However, we recommend against it, as it cannot be extended to model conditions at distances more than two. Also, we allow $k_{1}<k_{2}$, and their notation does not make it easy to tell which of $j$ and $k$ refers to $k_{1}$ or $k_{2}$.

Translating a labelling $f$, by adding the same element to all labels $f(v)$, preserves the span. Hence, to determine $\lambda(G ; \mathbf{k})$, it suffices to consider labellings with smallest label 0 . For such labellings $f, \operatorname{sp}(f)=\max _{v \in V} f(v)$.

Ordinary graph coloring corresponds to the case $k_{1}=1$ and $k_{i}=0$ otherwise. More precisely, the chromatic number $\chi(G)$ of a graph $G$ is expressed in our theory by

$$
\chi(G)=\lambda(G ; 1)+1,
$$

where the difference of one arises due to our allowing 0 to be a label. While it is convenient that $\lambda$ is one off from $\chi$, allowing 0 to be a label gives us the nice Scaling Property, in which if all separations $k_{i}$ are multiplied by the same constant $c$, then so is the optimal span $\lambda$. This principle is stated explicitly later when we formulate the theory of real number labellings.

An interesting special case, more general than above, is for $\mathbf{k}$ consisting of $p$ ones. Here we have

$$
\lambda(G ; 1, \ldots, 1)=\chi\left(G^{p}\right)-1,
$$

where $G^{p}$ is the graph that has the same vertex set $V$, and vertices $v, w$ are joined by an edge whenever their distance in $G$ is at most $p$.

Recent literature has expanded beyond the basic case of $L(2,1)$-labellings. Numerous papers consider $\lambda(G ; k, 1)$ for arbitrary integers $k>0$, or, more generally, $\lambda(G ; p, q)$ for integer separations $p \geq q$. 
Here is an overview of the rest of the paper. In the next section we introduce our model of labellings by real numbers. This more general setting is natural for many of the models in which labellings with distance conditions arise, since it would seem that the labels, e.g., frequencies, can actually be real numbers. Besides, we shall see that real number labellings offer greater insight into the behavior of the lambda number $\lambda(G ; \mathbf{k})$ when it is viewed as a function of the separations $\mathbf{k}=\left(k_{1}, \ldots, k_{p}\right)$ with fixed graph $G$ and separation distance $p$.

We also extend our model to allow infinite graphs $G$, such as the triangular lattice mentioned earlier. We will usually restrict our attention to infinite graphs with bounded degrees, to be assured of dealing with graphs of finite span. We denote by $\mathcal{G}_{\Delta}$ the class of simple graphs, possibly infinite, with maximum degree at most $\Delta$. In particular, $\Gamma_{\Delta} \in \mathcal{G}_{6}$.

Section 3 presents (without proof) our formulas for real number labellings with conditions at distance two for the triangular lattice, paths, cycles, and the square lattice. Their behavior is instructive and serves to motivate the results and conjectures in the rest of the paper.

Our main general discovery about real number labellings, the $D$-set Theorem, is presented in Section 4. It applies to the class of graphs $\mathcal{G}_{\Delta}$. It shows that $\lambda(G ; \mathbf{k})$ must be a sum of $k_{i}$ 's, repetitions allowed. Moreover, there is a labelling achieving $\lambda(G ; \mathbf{k})$ in which every label has the form $\sum_{i} a_{i} k_{i}$, where the coefficients $a_{i}$ are nonnegative integers, the smallest label is zero, and the largest label is the span. Further, if $G$ is finite, we can restrict the possible labels to those sums with $\sum_{i} a_{i}<n$, where $n=|V|$.

Among the consequences of the $D$-set Theorem is that when all $k_{i}$ are integers, our problem reduces to the familiar integer lambda labelling problem of Section 1: There is always an integer labelling that is optimal for the real number labelling problem.

In Section 5 we show that $\lambda(G ; \mathbf{k})$ is continuous as a function of the separations $\mathbf{k}$, and we conjecture that it is piecewise linear with only finitely many linear pieces. This is verified for separations at distance two $(p=2)$ and for finite graphs. Section 6 poses a stronger conjecture that there is always a labelling as in the $D$-set Theorem in which the coefficients are bounded above by a constant that depends only on $p$ and the degree bound $\Delta$. We can prove this for conditions out to distance two $(p=2)$.

Section 7 recalls the conjecture of [16] concerning the largest possible value of $\lambda(G ; 2,1)$ for graphs $G$ of maximum degree $\Delta \geq 2$. We survey the progress on this longstanding conjecture. We present upper bounds on $\lambda(G ; \mathbf{k})$ in terms of the separations $k_{i}$ and the maximum degree.

We describe work that is closely related to our project in Section 8. Besides the fascinating conjectures that remain open, the model of real number labellings has opened up interesting new lines of research, which we describe in the final section.

\section{From Integer to Real Number Labellings}

When the paper of Griggs and Yeh [16] introduced $\lambda$-labellings, it actually began with a special case of real number labellings, in which the transmitters were assigned real number labels and the separations were $\mathbf{k}=(2 d, d)$, where $d$ is some real number, not necessarily 
integer. It was shown that for any (finite) graph $G$, there is an optimal labelling in which all labels are multiples of $d$. But by a change of scale, dividing the separations and the labels by $d$, the problem was transformed into that of determining the optimal integer labelling span $\lambda(G ; 2,1)$. The same method applies to real labellings whenever the separations $k_{i}$ are multiples of the same real number $d$.

It has always seemed overly restrictive that channel assignment models assumed the channels-the vertex labels-are integers (or, equivalently, all are multiples of the same number). This is certainly the case for familiar VHF television, with its integer channels ranging from 2 to 13, but for FM radio and possibly even for UHF television, the channels appear to have a continuous range of possibilities. The efficient allocation of bands of the radio frequency spectrum is a subject receiving considerable publicity.

Reviewing the progress on $\lambda$-labellings over the years, it is now sensible to consider the generalization in which the separations $k_{i}$ and the labels $f(v)$ are arbitrary real numbers. As we would demand, this more general problem reduces to the familiar integer labellings when the separations and labels are integers. However, a rich variety of interesting new problems has been exposed by considering real number labellings, and their solution does not generally follow from their integer restrictions. Further, we have gained valuable new insights into the original integer $\lambda$-labellings by thinking in this more general context-see the $D$-set Theorem.

This study also widens the class of graphs to include infinite graphs, in order to be able to deal properly with infinite arrays of transmitters. For instance, in cellular communications, a very large flat area is partitioned honeycomb-style into hexagonal cells, with a transmitter located in the center of each cell (its coverage area). This transmitter placement is most efficient (minimizes the number of transmitters). The channel assignment for the transmitter network is equivalent to the lambda labelling of the vertices of the dual graph, where each vertex corresponds to a transmitter. Extending the cellular network over the whole plane, the dual graph is a planar graph in which every vertex has six neighbors, which form a cycle around the vertex. The regions of the embedding of the dual graph are all triangles. This infinite 6-regular graph is called the triangular lattice, which we denote by $\Gamma_{\Delta}$. Because of its potentially practical implications, the lambda labelling of $\Gamma_{\Delta}$ is of particular interest to us.

Let $G=(V, E)$ be any graph, possibly infinite. A real number labelling of $G$ is a function $f: V \rightarrow \mathbb{R}$, and its span is

$$
\operatorname{sp}(f):=\sup _{v \in V} f(v)-\inf _{v \in V} f(v) .
$$

We consider real separations $k_{1}, \ldots, k_{p} \in[0, \infty)$. Define $L(G ; \mathbf{k})=L\left(G ; k_{1}, \ldots, k_{p}\right)$ to be the set of real labellings $f: V \rightarrow \mathbb{R}$, such that

$$
|f(v)-f(w)| \geq k_{i}, \quad \text { if } d(v, w)=i \leq p .
$$

We may write $L(\mathbf{k})$ or $L\left(k_{1}, \ldots, k_{p}\right)$ if $G$ is understood.

We then define

$$
\lambda(G ; \mathbf{k}):=\inf _{f \in L(\mathbf{k})} \operatorname{sp}(f) .
$$


We will usually restrict our attention to the class $\mathcal{G}_{\Delta}$ of simple graphs with maximum degree at most $\Delta$. We shall see that there are labellings with bounded span in $L(G ; \mathbf{k})$ for $G \in \mathcal{G}_{\Delta}$, so $\lambda(G ; \mathbf{k})<\infty$ for all such $G$. Compactness arguments may be used to show that for $G \in \mathcal{G}_{\Delta}$, there is a labelling $f$ that actually achieves $\lambda(G ; \mathbf{k})$, meaning that its span is $\lambda(G ; \mathbf{k})$, and there are vertices on which $f$ assumes its minimum and maximum values, which are $\lambda(G ; \mathbf{k})$ apart (recall this is true for infinite graphs). However, we will derive this information by a simpler approach in the next section. Our approach will also provide information about possible values of $\lambda(G ; \mathbf{k})$.

As a simple introduction to real labellings, let us determine $\lambda\left(P_{3} ; k, 1\right)$, where $P_{3}$ is the path on three vertices and $k$ is any real $\geq 0$. One valid labelling is $(k, 0, k+1)$, and there is no way to improve on it among labellings where the middle label is the smallest of the three. If we put the smallest label on an end, and the largest is in the middle, we can do no better than $(0, k+1,1)$. If the smallest and largest labels are at the ends, we can either use $(0, k, 2 k)$, provided that $k \geq 1 / 2$, or $(0, k, 1)$, when $k \leq 1 / 2$.

It follows that

$$
\lambda\left(P_{3} ; k, 1\right)=\left\{\begin{array}{lll}
1, & \text { if } & 0 \leq k \leq 1 / 2 \\
2 k, & \text { if } & 1 / 2 \leq k \leq 1 \\
k+1, & \text { if } & 1 \leq k
\end{array}\right.
$$

Notice that we are allowing $k_{1}=k$ to be less than $k_{2}=1$, which seems strange at first. Indeed, in models of interference between nearby transmitters, one expects the interference to decrease with distance, so that the required separations $k_{i}$ would be nonincreasing as $i$ grows. However, some recent papers have considered situations where the $k_{i}$ may increase with $i$, and our model allows arbitrary $k_{i} \geq 0$ (which is mathematically interesting regardless of its usefulness).

We mention how it can arise in practice that $k_{1}<k_{2}$. Jin and Yeh [20] cites a packet communication model of Bertossi and Barnstormer [2] that considers such a case. Message packets are being sent throughout a wireless network of computer stations (computers and transceivers). The computer stations are the vertices and wireless connections between them are the edges if they can hear each other due to their proximity. Using the Code Division Multiple Access protocol (CDMA), each computer station is assigned a control code, and packets are sent along the edges simultaneously, using the control codes of the computer stations sending them. These codes correspond to channels in our model. A problem arises whenever a computer station receives packets simultaneously from two different adjacent computer stations that cannot hear each other and use the same control code, and the receiving computer station has to ask for the packets to be resent. Avoiding this interference then requires that no two computer stations at distance two in the network use the same control code. Minimizing the number of different control codes used is then the $L(0,1)$ problem for the corresponding graph.

Of course, this is really just a standard graph coloring problem in disguise, but for a different graph: Given $G=(V, E)$, we form the graph $G^{\prime}=\left(V, E^{\prime}\right)=\left(G^{2}-G\right)$, in which $E^{\prime}=E\left(G^{2}\right)-E(G)$ contains pairs of vertices that are at distance two in $G$. Then $\lambda(G ; 0,1)=\chi\left(G^{\prime}\right)-1$. It would be interesting to find other situations that require lambda 
labellings $L\left(k_{1}, \ldots, k_{p}\right)$ in which $k_{i}<k_{j}$ for some $i<j$.

Returning to the path $P_{3}$, we have already given the values $\lambda\left(P_{3} ; k, 1\right)$. Let us note also that $\lambda\left(P_{3} ; 1,0\right)=\chi\left(P_{3}\right)-1=1$. We can now obtain all lambda numbers for the path $P_{3}$ with conditions at distance two from the following principle:

Property 2.1 (Scaling Property) For all reals $k_{1}, \ldots, k_{p}, c \geq 0$ and all graphs $G$,

$$
\lambda\left(G ; c k_{1}, \ldots, c k_{p}\right)=c \lambda\left(G ; k_{1}, \ldots, k_{p}\right) .
$$

This Property is an immediate consequence of the definition of $\lambda$, since if any labelling of $G$ with separation conditions $\mathbf{k}$ has its labels each multiplied by $c$, it gives a labelling with separation conditions $c \mathbf{k}$, and vice-versa.

For conditions at distance two, the Scaling Property gives us that

$$
\lambda\left(G ; k_{1}, k_{2}\right)=k_{2} \lambda(G ; k, 1)
$$

for $k=k_{1} / k_{2}, k_{2}>0$. So we can derive all values of $\lambda$ with $k_{2}>0$ from the one-parameter values $\lambda(G ; k, 1), k \geq 0$, such as we gave above for $G=P_{3}$. We can also obtain the values $\lambda\left(G ; k_{1}, 0\right)$, which is given by $k_{1} \lim _{k \rightarrow \infty}(\lambda(G ; k, 1) / k)$.

We next give a simple general upper bound on $\lambda(G ; \mathbf{k})$ in terms of the maximum degree and the separations $k_{i}$ for use in the proofs. Better bounds are given later in Section 8 .

Lemma 2.2 Let $G$ be a graph, possibly infinite, of maximum degree at most $\Delta$. Let $p \in \mathbb{Z}^{+}, \mathbf{k}=\left(k_{1}, \ldots, k_{p}\right)$, and $k=\max _{i}\left\{k_{i}\right\}$. Then $\lambda(G ; \mathbf{k}) \leq k \Delta^{p}$.

Proof. Let $G \in \mathcal{G}_{\Delta}$. For such $p$ and $\mathbf{k}$ we have that

$$
\lambda(G ; \mathbf{k}) \leq \lambda(G ; k, \ldots, k)=k \lambda(G ; 1, \ldots, 1)=k\left(\chi\left(G^{p}\right)-1\right),
$$

which is, in turn, at most $k$ times the maximum degree of graph $G^{p}$. Since $G$ has at most $\Delta(\Delta-1)^{i-1}$ vertices at distance $i$ from any given vertex, we get that the maximum degree of $G^{p}$ is at most

$$
\Delta \sum_{i=1}^{p}(\Delta-1)^{i-1} \leq \Delta^{p}
$$

Note that a labelling that satisfies the bound of Lemma 2.2 can be obtained by first arbitrarily ordering the vertices in some component of $G^{p}$. One can then greedily color the vertices in the component one-by-one in order by nonnegative integers, always selecting the least color not already assigned to any neighboring vertex. Do this for each component. (This is a so-called first-fit labelling.) Then multiply all the labels by $k$ to obtain a suitable labelling in $L(G ; \mathbf{k})$. 


\section{Optimal Spans with Conditions at Distance Two for Special Graphs}

In order to motivate the $D$-set Theorem and other general results in later sections concerning the general behavior of $\lambda(G ; \mathbf{k})$ viewed as a function of $\mathbf{k}$, we present some of our findings for particular graphs. Some of the results in this section were obtained, in part, by using the $D$-set Theorem. But many of them were obtained independently of it, before the discovery of the $D$-set Theorem, and played a role in its discovery. We postpone the long and intricate proofs with many cases to later papers $[15,14]$.

We consider only conditions at distance at most two. As noted above, it suffices to determine $\lambda(G ; k, 1)$. There are various results in the literature concerning $\lambda(G ; k, 1)$ when $k$ is a positive integer. In the 2000 Mathematical Competition in Modeling ("MCM"), a problem of this kind (written by Griggs for the contest) was presented. The problem can be found in the special journal issue for the contest that includes a survey article by Griggs [12], or on the Web at www.comap.com. The problem was selected and reported on by 271 teams, each consisting of three undergraduates, from universities worldwide. Each team had a long weekend (less than four days) to research the problem, write and run programs, and put together a paper. They had access to libraries, computers, and the Web, but no human assistance was permitted.

Teams were asked in this problem to investigate distance labellings of the triangular lattice graph $\Gamma_{\Delta}$. In some cellular communication networks [22] a large planar region is partitioned into hexagonal cells with a transmitter at the center of each cell. This method gives efficient coverage (minimizes the number of transmitters needed). Strong interference occurs between transmitters in adjacent cells, while lighter interference occurs between transmitters in cells with just one cell in between. We may form a graph, with a vertex for each cell and an edge between each two vertices that represent adjacent cells. In this case, we are fortunate in that the graph labelling problem with conditions at distance two is actually equivalent to the original transmitter problem in the plane. When the planar coverage region is the entire plane, the corresponding graph is an infinite 6-regular graph, the triangular lattice, which we denote by $\Gamma_{\Delta}$. MCM teams were asked to determine $\lambda(G ; 2,1)$ for $G$ corresponding to a certain large region and then for the entire plane (for $G=\Gamma_{\Delta}$ ). While experts in the subject already knew the (unpublished) answer, it was pleasing to see how many teams succeeded. MCM teams were asked to determine what they could about $\lambda\left(\Gamma_{\Delta} ; k, 1\right)$ for integers $k>1$. Several teams devised labellings that turned out to be optimal, though no team came up with a valid proof for general $k$ : Their lower bound proofs were not adequate. Condensed versions of the winning papers are collected in the special UMAP journal issue mentioned above [12].

A subsequent manuscript of Zhu and Shi [30] considers $\lambda\left(\Gamma_{\Delta} ; k_{1}, k_{2}\right)$ for general integers $k_{1} \geq k_{2} \geq 1$. It provided more impetus to undertake the study contained in this paper. Note that by scaling, we shall find that to determine $\lambda\left(\Gamma_{\Delta} ; k_{1}, k_{2}\right)$ for such integers is equivalent to determining $\lambda\left(\Gamma_{\Delta} ; k, 1\right)$ for rationals $k \geq 1$.

With considerable effort, the present authors have completely determined the values $\lambda\left(\Gamma_{\Delta} ; k, 1\right)$ for all reals $k \geq 1$. For reals $0 \leq k<1$, we have been chipping away, 
determining the exact value for small $k$ and for some other values $k$, and bounds otherwise:

Theorem 3.1 [14] For the triangular lattice $\Gamma_{\Delta}$, we have the following values (or bounds, where it is not yet determined) for optimal spans of labellings with conditions at distance two:

$$
\lambda\left(\Gamma_{\Delta} ; k, 1\right)=\left\{\begin{array}{lll}
2 k+3, & \text { if } \quad 0 \leq k \leq 1 / 3 ; \\
\in[2 k+3,11 k], & \text { if } \quad 1 / 3 \leq k \leq 9 / 22 ; \\
\in[2 k+3,9 / 2], & \text { if } \quad 9 / 22 \leq k \leq 3 / 7 \\
\in[9 k, 9 / 2], & \text { if } \quad 3 / 7 \leq k \leq 1 / 2 ; \\
\in[9 / 2,16 / 3], & \text { if } \quad 1 / 2 \leq k \leq 2 / 3 ; \\
\in[16 / 3,23 / 4], & \text { if } \quad 2 / 3 \leq k \leq 3 / 4 ; \\
\in[23 / 4,6], & \text { if } \quad 3 / 4 \leq k \leq 4 / 5 ; \\
6, & \text { if } \quad 4 / 5 \leq k \leq 1 ; \\
6 k, & \text { if } \quad 1 \leq k \leq 4 / 3 ; \\
8, & \text { if } \quad 4 / 3 \leq k \leq 2 ; \\
4 k, & \text { if } \quad 2 \leq k \leq 11 / 4 ; \\
11, & \text { if } \quad 11 / 4 \leq k \leq 3 ; \\
3 k+2, & \text { if } \quad 3 \leq k \leq 4 ; \\
2 k+6, & \text { if } \quad k \geq 4 ;
\end{array}\right.
$$

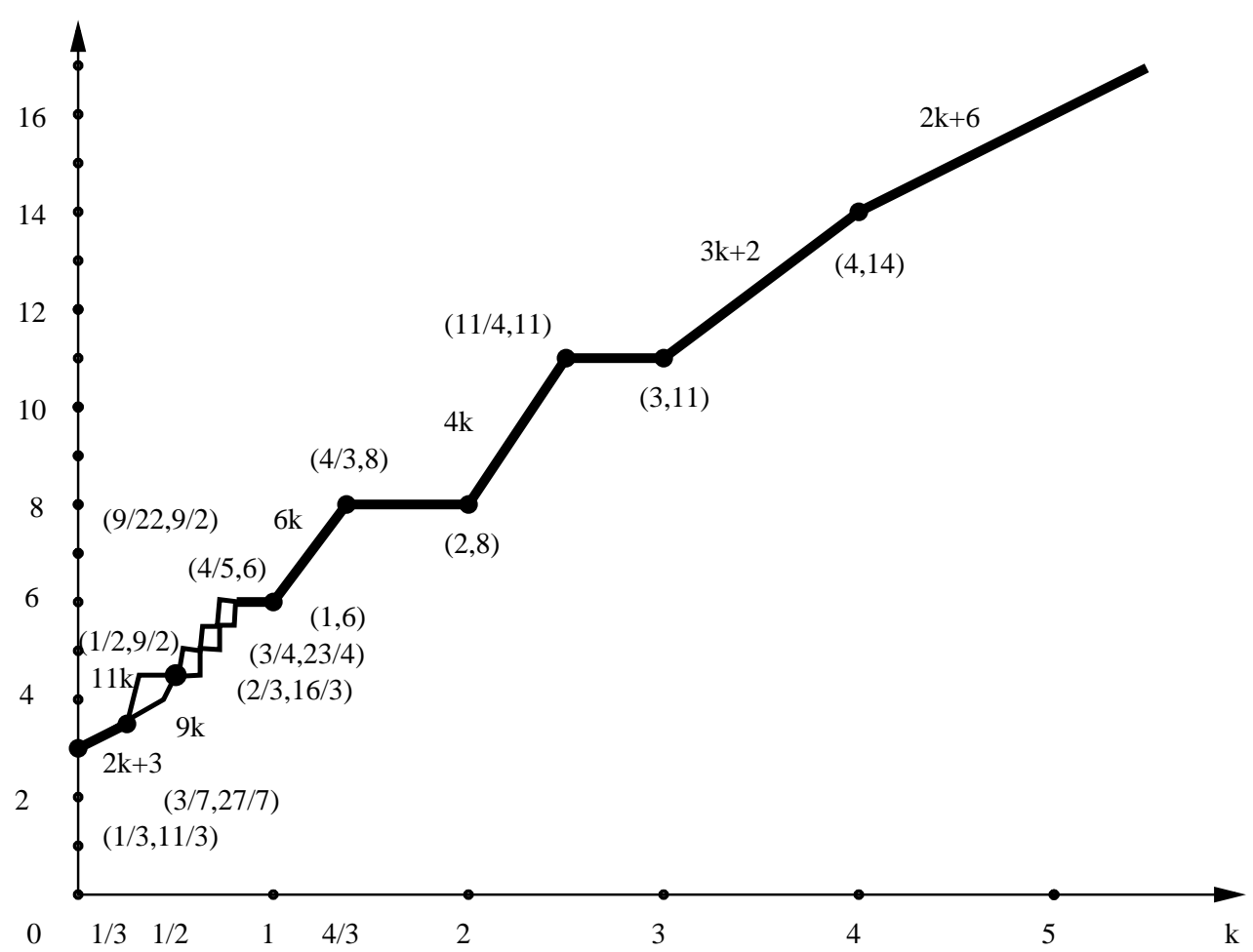

Figure 3: A graph of $\lambda\left(\Gamma_{\triangle} ; k, 1\right)$ as a function of $k$. 
A graph of $\lambda\left(\Gamma_{\Delta} ; k, 1\right)$ as a function of $k$ is presented in Figure 3. Coordinates are given for endpoints and isolated points that are known precisely. For $k \geq 1$ the graph is seen to be nondecreasing, continuous, and piecewise linear, and the same appears likely for $k \leq 1$. Curiously, it is neither convex nor concave, nor is it even strictly increasing (at least three sections are flat). It will follow from Theorem 5.6 that the graph is piecewise linear over its whole domain, $[0, \infty)$, even though we cannot yet give it on $[0,1]$.

We have completely determined $\lambda(G ; k, 1)$ for paths $P_{n}$ and cycles $C_{n}$ on $n$ vertices:

Theorem 3.2 [15] For the paths $P_{n}$, the optimal span $\lambda\left(P_{n} ; k, 1\right)$ with conditions at distance two, for $k \geq 0$, is shown in Figure 4. In particular, the optimal span is the same for all $k$ for $n \geq 7$.

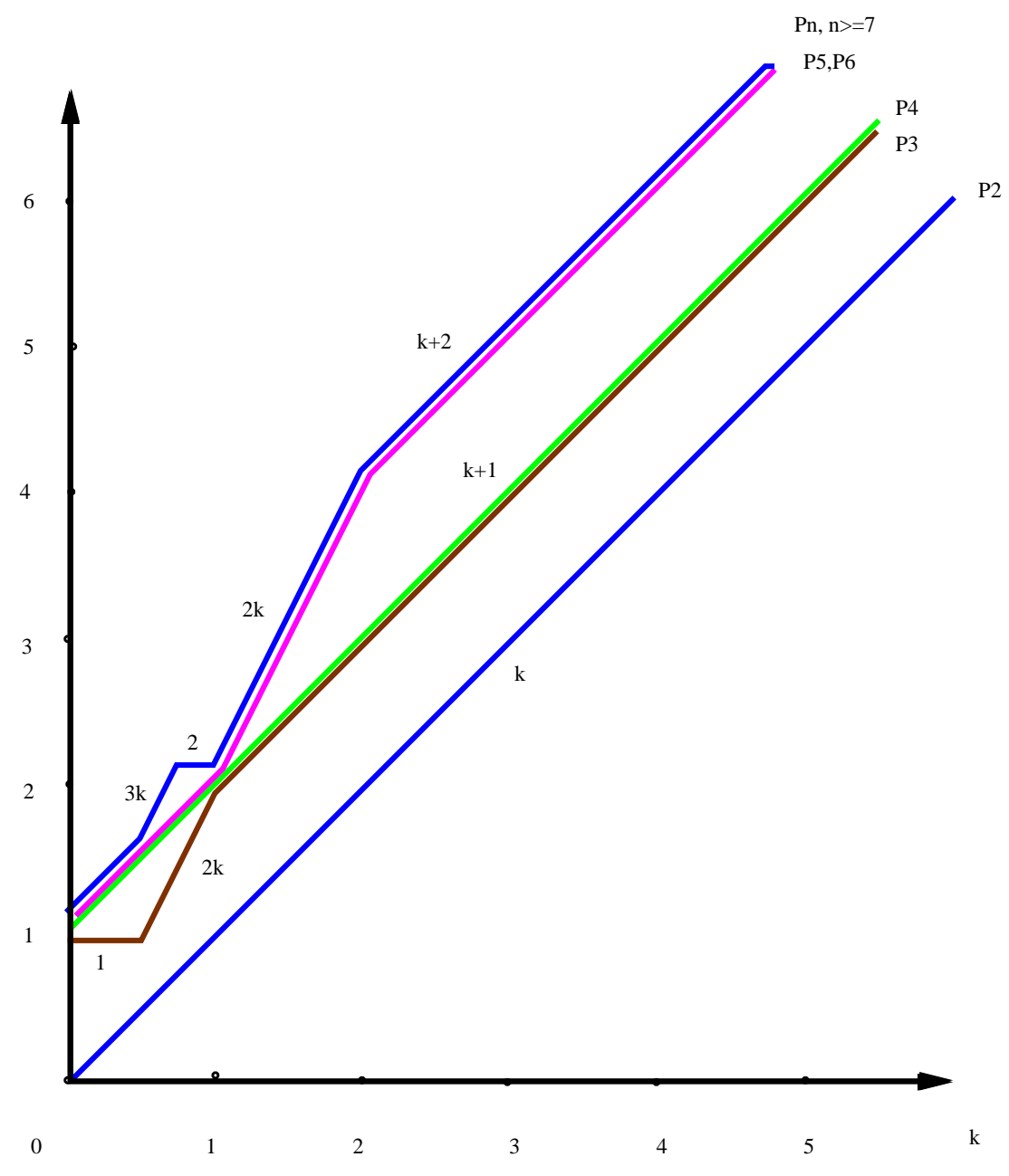

Figure 4: The functions $\lambda\left(P_{n} ; k, 1\right)$ for the paths $P_{n}$ on $n$ vertices.

Theorem 3.3 [15] For the cycles $C_{n}$, the optimal span $\lambda\left(C_{n} ; k, 1\right)$ with conditions at distance two, for $k \geq 0$, is shown in Figure 5 for $n=3,4,5$ and in Figure 6 for $n \geq 6$. 


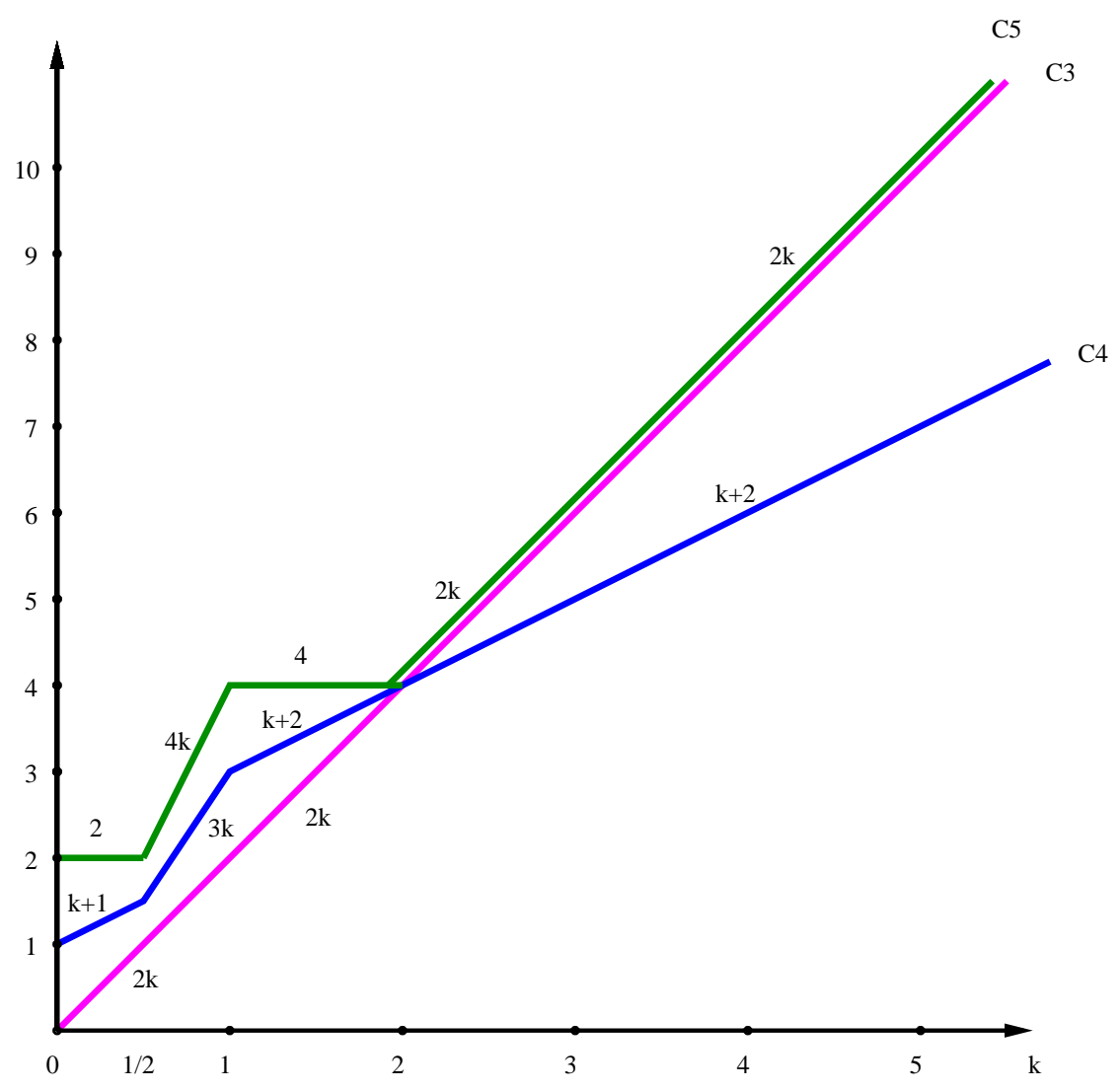

Figure 5: The functions $\lambda\left(C_{n} ; k, 1\right)$ for the cycles $C_{n}, n=3,4,5$.

Note: For $n \geq 6$, the choice of curve to follow in Figure 6 depends on the value of $n$ modulo 12. For instance, in the interval $[2 / 3,2]$, one follows the lower piece when $n$ is $0(\bmod 12)$ and the upper piece when $n$ is $1(\bmod 12)$.

After obtaining these path and cycle lambda number formulas, we realized that they were already known in part: Georges and Mauro [8] determined them for integer separations $k_{1} \geq k_{2}$. By the Scaling Property their formulas give $\lambda(G ; k, 1)$ for rationals $k \geq 1$ when $G$ is a path or cycle (see Section 8 for related remarks).

Notice that for each path and cycle, the graph is again a continuous nondecreasing piecewise linear function. Also, the linear formulas for the straight sections of the graphs above are always of the form $a k+b$, where $a$ and $b$ are nonnegative integers. For the graph $\Gamma_{\Delta}$, one of the winning teams in the Modeling Contest, from Washington University [11] claimed that this should be the case for the triangular lattice for all (integers) $k$. They turned out to be correct. Indeed, we shall see there is a piecewise linearity result, where the pieces are nonnegative integer linear functions of $k_{1}$ and $k_{2}$, for general graphs of bounded degree (Theorem 5.6).

We wish to state one more important example, the square lattice $\Gamma_{\square}$, which is used in some applications. Here, the vertices correspond to the integer lattice points in the plane, 


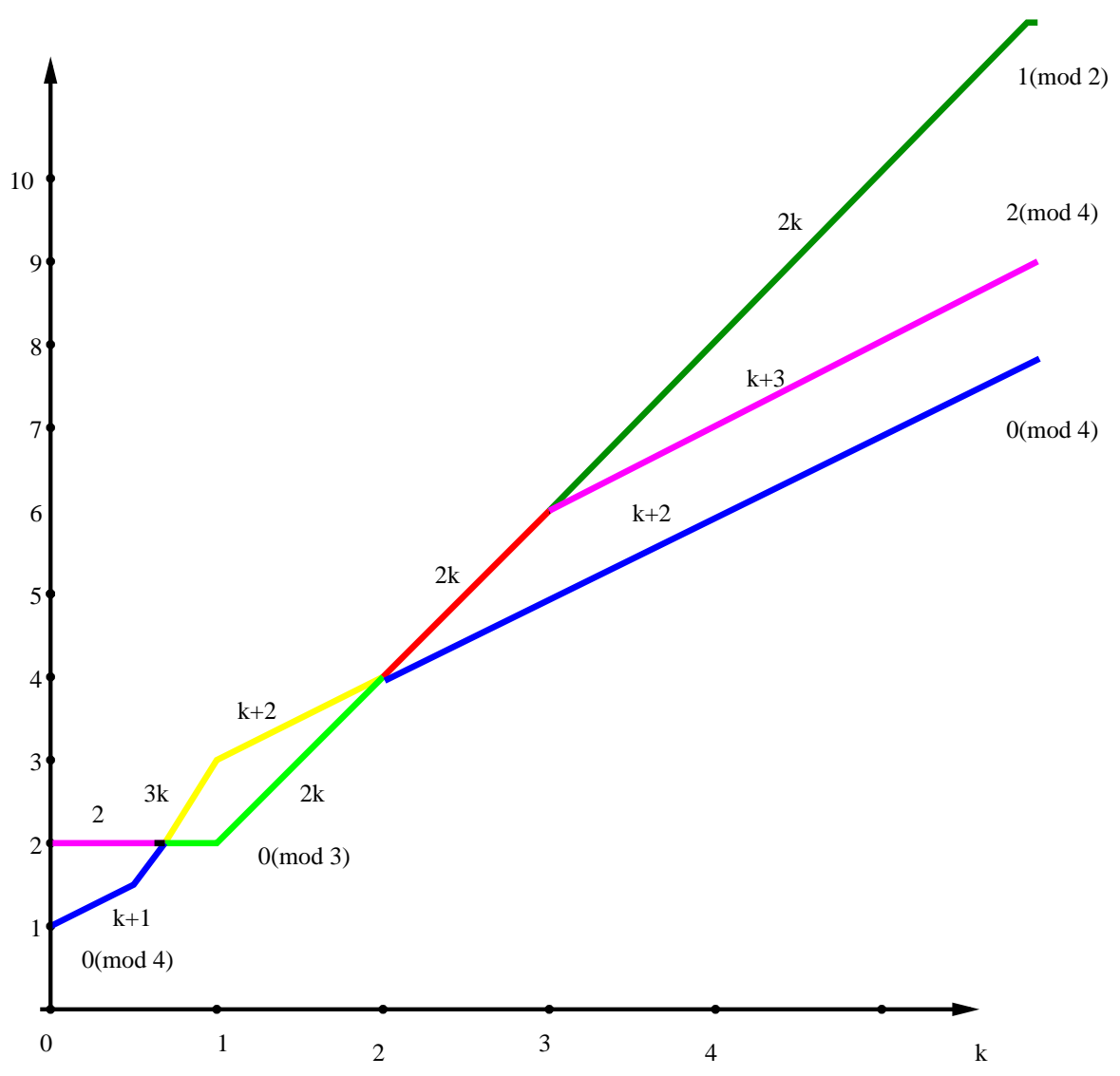

Figure 6: The functions $\lambda\left(C_{n} ; k, 1\right)$ for the cycles $C_{n}, n \geq 6$.

and edges join pairs of vertices that are equal in one coordinate and are consecutive in the other coordinate. It is possible to give the complete formula for labellings with conditions at distance two, and, as expected, it is piecewise linear with finitely many pieces. The graph of $\lambda\left(\Gamma_{\square} ; k, 1\right)$ is shown in Figure 7 . 
Theorem 3.4 [14] For the square lattice $\Gamma_{\square}$ we have the following values for optimal spans of labellings with conditions at distance two:

$$
\lambda\left(\Gamma_{\square} ; k, 1\right)=\left\{\begin{array}{lll}
k+3, & \text { if } \quad 0 \leq k \leq 1 / 2 ; \\
7 k, & \text { if } \quad 1 / 2 \leq k \leq 4 / 7 ; \\
4, & \text { if } \quad 4 / 7 \leq k \leq 1 ; \\
4 k, & \text { if } 1 \leq k \leq 4 / 3 ; \\
k+4, & \text { if } 4 / 3 \leq k \leq 3 / 2 ; \\
3 k+1, & \text { if } 3 / 2 \leq k \leq 5 / 3 ; \\
6, & \text { if } 5 / 3 \leq k \leq 2 ; \\
3 k, & \text { if } 2 \leq k \leq 8 / 3 ; \\
8, & \text { if } 8 / 3 \leq k \leq 3 ; \\
2 k+2, & \text { if } 3 \leq k \leq 4 ; \\
k+6, & \text { if } \quad k \geq 4 .
\end{array}\right.
$$

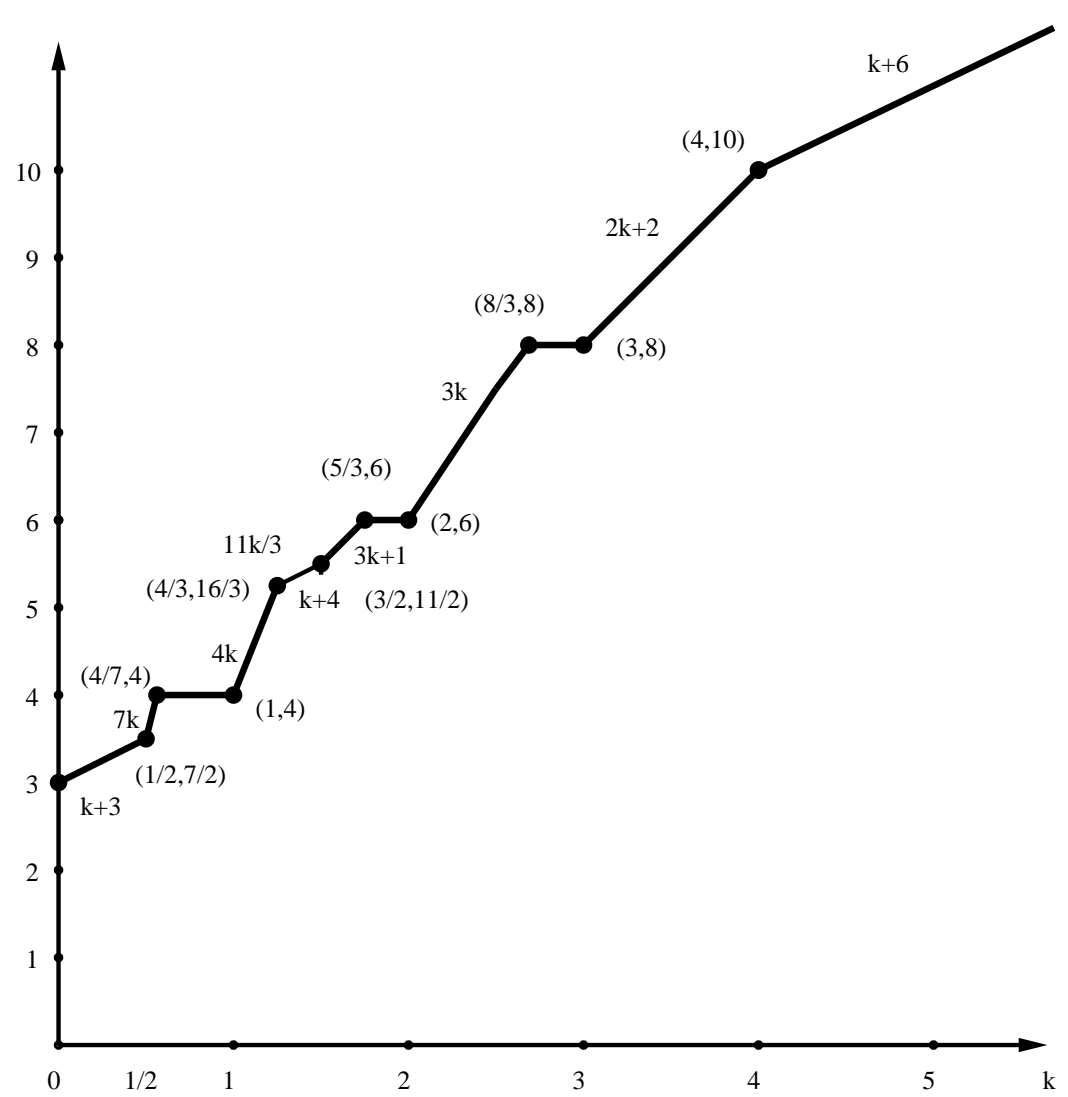

Figure 7: A graph of $\lambda\left(\Gamma_{\square} ; k, 1\right)$ as a function of $k$. 


\section{$4 \quad D$-set Theorem on Real Labellings}

Motivated by partial results for paths and cycles (our complete solution came later) and by observations described in the previous section, we came to realize that for any finite graph $G$ and any fixed separation vector $\mathbf{k}=\left(k_{1}, \ldots, k_{p}\right), \lambda(G ; \mathbf{k})$ must be a sum of the separations $k_{i}$ (repeats allowed). Indeed, this holds as well for the infinite graph $\Gamma_{\Delta}$ (as claimed by the MCM team from Washington University), and, in general, for any graph with bounded degrees.

Given k, let us define the " $D$-set"

$$
D\left(k_{1}, \ldots, k_{p}\right):=\left\{\sum_{i=1}^{p} a_{i} k_{i}: a_{i} \in \mathbb{N}, 1 \leq i \leq p\right\},
$$

abbreviated by $D(\mathbf{k})$. It turns out that it suffices to consider labellings with labels in $D(\mathbf{k})$ in order to prove the existence of optimal $\lambda$-labellings:

Theorem 4.1 (D-set Theorem) Let $G=(V, E)$ be a graph, possibly infinite, of bounded maximum degree. Let $k_{1}, \ldots, k_{p}$ be real numbers $\geq 0$. Then there is an optimal labelling $f \in L(\mathbf{k})$ with all labels $f(v) \in D(\mathbf{k})$ in which the smallest label is 0 , the largest label is $\lambda(G ; \mathbf{k})$. In particular, $\lambda(G ; \mathbf{k}) \in D(\mathbf{k})$. Moreover, if $G$ is finite, each label $f(v)$ and $\lambda(G ; \mathbf{k})$ can be expressed in the form $\sum_{i} a_{i} k_{i}$, where the nonnegative integer coefficients $a_{i}$ satisfy $\sum_{i} a_{i}<|V|$.

Proof. Let $\mathbf{k}=\left(k_{1}, \ldots, k_{p}\right)$, and suppose $G$ is a graph in $\mathcal{G}_{\Delta}$. Let $f \in L(\mathbf{k})$ be any labelling of span at most $\Delta^{p} k$, where $k=\max _{i}\left\{k_{i}\right\}$; such labellings exist by Lemma 2.2. By translating the labelling $f$, if necessary, we may assume that $\inf _{v} f(v)=0$. We modify $f$ to get another labelling $f^{*}$, with span no larger than for $f$, such that all labels for $f^{*}$ belong to $D(\mathbf{k})$. Also, $f^{*}$ will have smallest label 0 .

Let us define the $D$-floor of a real number $x \geq 0$ by

$$
\lfloor x\rfloor_{D}:=\max \{y \in D(\mathbf{k}): y \leq x\} .
$$

Note that $D(\mathbf{k})$ contains only a finite number of elements $\leq x$, so this is a maximum, not a supremum. Then we define the new labelling $f^{*}$ at each vertex $v$ by

$$
f^{*}(v):=\lfloor f(v)\rfloor_{D}
$$

Because $D(\mathbf{k})$ has only finitely many elements in $\left[0, \Delta^{p} k\right]$, and $\inf _{v} f(v)=0$, it follows that $f^{*}$ has minimum value 0 . By design, all values $f^{*}(v)$ belong to $D(\mathbf{k})$. It suffices to show that $f^{*} \in L(\mathbf{k})$, which requires checking the separation conditions. Suppose that $u, v \in V$ are at distance $i \leq p$ in $G$. Without loss of generality, suppose $f(u) \geq f(v)$. Since $f \in L(\mathbf{k})$, we find that

$$
f(u) \geq f(v)+k_{i} \geq\lfloor f(v)\rfloor_{D}+k_{i}=f^{*}(v)+k_{i} .
$$


Since $f^{*}(v)+k_{i} \in D(\mathbf{k})$, it follows from the definition of the $D$-floor that $\lfloor f(u)\rfloor_{D} \geq$ $f^{*}(v)+k_{i}$, so that

$$
f^{*}(u) \geq f^{*}(v)+k_{i},
$$

and the separation conditions are satisfied.

We have shown that for every $f \in L(\mathbf{k})$, there exists $f^{*} \in L(\mathbf{k})$ with $\operatorname{sp}\left(f^{*}\right) \leq \operatorname{sp}(f)$ such that $\operatorname{sp}\left(f^{*}\right) \leq C$ and $\operatorname{sp}\left(f^{*}\right) \in D(\mathbf{k})$. Since $D(\mathbf{k}) \cap[0, C]$ is finite, it follows that $\lambda$ exists, belongs to $D(\mathbf{k})$, and is at most $C$. Also, there exists an optimal labelling where the labels are in $D(\mathbf{k})$, with smallest label 0 and largest label $\lambda(G ; \mathbf{k})$.

If $G$ has $n<\infty$ vertices, let $f$ be an optimal labelling of $G$ as above. The minimum label $f(v)$ is 0 , say it occurs at $v_{1}, \ldots, v_{s}$. Consider the smallest label $>0$, say it occurs at $v_{s+1}$. We may decrease $f\left(v_{s+1}\right)$ to 0 without any problem, and repeat this process, unless there is some $t<s+1$ such that $v_{t}$ and $v_{s+1}$ are at some distance $d$ with $k_{d}>0$. But we may at least still decrease $f\left(v_{s+1}\right)$ until it is some value $k_{i} \geq k_{d}>0$, where some vertex $v_{r}$ is at distance $i$ from $v_{s+1}$, with $r<s+1$.

Then we decrease the next smallest label until it is a sum of at most two $k_{i}$ 's, not necessarily distinct, and so on, until all labels are sums of fewer than $n k_{i}$ 's. In doing this, labels only get smaller or remain unchanged. Thus, in the end we still have an optimal labelling, and its span is a sum of fewer than $n k_{i}$ 's, not necessarily distinct.

We now confirm that real number labellings include integer labellings as a special case:

Corollary 4.2 Let $G=(V, E)$ be a graph, possibly infinite, of bounded maximum degree. Let $k_{1}, \ldots, k_{p}$ be integers $\geq 0$. Then $\lambda(G ; \mathbf{k})$ is an integer, and there is an optimal labelling with smallest label 0 and every label integer.

Example 4.3 Suppose $G$ is a graph with bounded maximum degree, and suppose $\mathbf{k}=$ $(5,3)$. Then there is an optimal labelling with smallest label 0 and all labels, and $\lambda(G ; 5,3)$, belonging to $D(5,3)=\{0,3,5,6,8,9,10, \ldots\}$. In particular, to search for $\lambda(G ; 5,3)$, it suffices to try nonnegative integer labellings, with smallest label 0 , that do not use 1, 2, 4, or \%. This could speed up computing $\lambda(G ; 5,3)$.

The $D$-set Theorem is particularly useful for proving lower bounds on $\lambda(G ; \mathbf{k})$. (Explicit labellings are used to prove upper bounds.) Here are two proofs of (sharp) lower bounds for paths that rely on the $D$-set Theorem.

Example 4.4 For $1 / 2 \leq k \leq 1$, we have $\lambda\left(P_{3} ; k, 1\right) \geq 2 k$.

Proof. Suppose not, say $\lambda\left(P_{3} ; k, 1\right)<2 k$. This forces $k>1 / 2$. By the $D$-set Theorem, there is an optimal labelling $f$ using labels only in $[0,2 k) \cap D(k, 1)=\{0, k, 1\}$. For $P_{3}$, the labels must be distinct by the separation conditions. But even that is impossible, since label $k$ is too close to 1 : Their difference, $1-k$ is less than $k$ and 1 . So no such $f$ exists.

Example 4.5 For $1 / 2 \leq k \leq 2 / 3$ (resp., $2 / 3 \leq k \leq 1), \lambda\left(P_{7} ; k, 1\right) \geq 3 k$ (resp., $\geq 2$ ). 
Proof. For this range of $k$ values we have the smallest elements of $D(k, 1)$ are, in order, $0, k, 1,2 k, k+1$, followed by $3 k$ (resp., 2), if $1 / 2 \leq k \leq 2 / 3$ (resp., $2 / 3 \leq k \leq 1$ ). By the $D$-set Theorem, it suffices to show that $\lambda\left(P_{7} ; k, 1\right)>k+1$ for $1 / 2<k \leq 1$. Suppose not, say $f$ is a $L(k, 1)$-labelling of $P_{7}$ using labels in the set $\{0, k, 1,2 k, k+1\}$.

Vertices not labelled with 0 have labels in $[k, k+1]$. Since $P_{3}$ has minimum span $2 k>1$ by Example 4.4, $f$ cannot assign nonzero labels to three consecutive vertices. But $f$ cannot assign 0 to two vertices at distance two or less. Hence, 0 labels must be used at some vertices at distance a multiple of three. If three labels are zeros, they are at the middle vertex and both endpoints of $P_{7}$, then all other labels are at least one, and one of them is at least two, which is larger than the span, a contradiction. If two labels are zeros, neither of them is at the endpoints of $P_{7}$. The two nonzero labels in between are both at least one, and at least $k$ apart from each other, so they must be 1 and $k+1$. Then the nonzero label at distance two from label 1 is at least 2, which is larger than the span, a contradiction.

\section{$5 \quad$ Piecewise Linearity}

For a fixed graph $G$ and a fixed integer $p$, we wish to understand the behavior of the optimal span $\lambda$ as a function of the minimum separations $k_{1}, \ldots, k_{p}$ in the set

$$
T^{p}=\left\{\left(k_{1}, \ldots, k_{p}\right) \in \mathbb{R}^{p}: k_{i} \geq 0 \forall i\right\}
$$

In particular, we want to see why it is piecewise linear in all examples we have studied.

We first obtain the continuity of $\lambda$ on $T^{p}$, which ends up being unexpectedly tricky to prove near the boundary.

Theorem 5.1 Let $G=(V, E)$ be a graph, possibly infinite, of bounded maximum degree. Let $p \in \mathbb{Z}^{+}$. Then $\lambda(G ; \mathbf{k})$ is continuous and nondecreasing as a function of $\mathbf{k}$ on $T^{p}$.

Proof. Let $G \in \mathcal{G}_{\Delta}$. For any $\mathbf{k}=\left(k_{1}, \ldots, k_{p}\right), \mathbf{k}^{\prime}=\left(k_{1}^{\prime}, \ldots, k_{p}^{\prime}\right) \in T_{p}$, we write $\mathbf{k} \leq \mathbf{k}^{\prime}$ if $k_{i} \leq k_{i}^{\prime}$ for all $i$. The function $\lambda$ is nondecreasing, since if $\mathbf{k} \leq \mathbf{k}^{\prime}$, the separations $\mathbf{k}^{\prime}$ are more restrictive than $\mathbf{k}$, so that $L\left(\mathbf{k}^{\prime}\right) \subseteq L(\mathbf{k})$, and thus $\lambda(G ; \mathbf{k}) \leq \lambda\left(G ; \mathbf{k}^{\prime}\right)$.

We show that $\lambda(G ; \mathbf{k})$ is continuous at an arbitrary $\mathbf{k} \in T^{p}$. Let $I$ be the set of indices $i$ where $k_{i}>0$. Let $\mathbf{k}^{*}$ be any element of $T_{p}$ that is distance at most $\varepsilon>0$ from $\mathbf{k}$. We need to show that $\left|\lambda\left(G ; \mathbf{k}^{*}\right)-\lambda(G ; \mathbf{k})\right|$ can be made arbitrarily small by selecting $\varepsilon$ small enough. Assume $\varepsilon<\left(\min _{i \in I} k_{i}\right) / 2$. Define vectors $\mathbf{k}^{\prime}, \mathbf{k}^{\prime \prime} \in T^{p}$, where $\mathbf{k}^{\prime \prime} \leq \mathbf{k} \leq \mathbf{k}^{\prime}$, as follows: Let $k_{i}^{\prime}=k_{i}+\varepsilon$ for all $i$, while $k_{i}^{\prime \prime}=k_{i}-\varepsilon$ for $i \in I$ and $k_{i}^{\prime \prime}=k_{i}=0$ otherwise. By design, $\mathbf{k}^{\prime \prime} \leq \mathbf{k}^{*} \leq \mathbf{k}^{\prime}$. As $\lambda$ is nondecreasing, we have that

$$
\left|\lambda\left(G ; \mathbf{k}^{*}\right)-\lambda(G ; \mathbf{k})\right| \leq \lambda\left(G ; \mathbf{k}^{\prime}\right)-\lambda\left(G ; \mathbf{k}^{\prime \prime}\right),
$$

and it suffices to show that $\lambda\left(G ; \mathbf{k}^{\prime}\right)-\lambda\left(G ; \mathbf{k}^{\prime \prime}\right)$ can be made arbitrarily small as $\varepsilon \rightarrow 0$.

Let $f^{\prime \prime}$ be an optimal labelling as in the $D$-set Theorem achieving $\lambda\left(G ; \mathbf{k}^{\prime \prime}\right)$. We will modify $f^{\prime \prime}$ to obtain a labelling $f^{\prime} \in L\left(\mathbf{k}^{\prime}\right)$ with span only slightly larger. Specifically, 
since $\lambda\left(G ; \mathbf{k}^{\prime}\right)-\lambda\left(G ; \mathbf{k}^{\prime \prime}\right) \leq \operatorname{sp}\left(f^{\prime}\right)-\operatorname{sp}\left(f^{\prime \prime}\right)$, it will suffice that $\operatorname{sp}\left(f^{\prime}\right)-\operatorname{sp}\left(f^{\prime \prime}\right) \rightarrow 0$ as $\varepsilon \rightarrow 0$. Since $0 \leq k_{i}^{\prime}-k_{i}^{\prime \prime} \leq 2 \varepsilon$ for all $i$, $f^{\prime}$ will be feasible for separations $\mathbf{k}^{\prime}$, if, for each pair of vertices at distance at most $p$ in $G, f^{\prime}$ increases the separation between their labels by at least $2 \varepsilon$.

By the $D$-set Theorem, all labels used by $f^{\prime \prime}$ belong to the set $D\left(\mathbf{k}^{\prime \prime}\right) \cap\left[0, \lambda\left(G ; \mathbf{k}^{\prime \prime}\right)\right]$. Let us denote these labels by $0=r_{1}<r_{2}<\cdots r_{A}$. Each of these labels $r_{j}$ has the form $\sum_{i \in I} a_{i} k_{i}^{\prime \prime}$, where the coefficients $a_{i}$ are integers $\geq 0$. Now we have

$$
a_{i}\left(k_{i} / 2\right) \leq a_{i} k_{i}^{\prime \prime} \leq \lambda\left(G ; \mathbf{k}^{\prime \prime}\right) \leq \lambda(G ; \mathbf{k}) \leq \Delta^{p} k,
$$

where we use Lemma 2.2. Hence, for all $i \in I, a_{i} \leq 2 C / k_{i}$, so that $a_{i}$ is bounded in terms of $\mathbf{k}$ and $\Delta$. It follows that the number of labels used by $f^{\prime \prime}, A$, is bounded in terms of $\mathbf{k}$ and $\Delta$.

Now we modify the labels $r_{i}$ two different ways. Let $\delta$ be a small number, depending on $\varepsilon$. First, increase each label $r_{i}$ by $(i-1) \delta$, which increases the separation between each pair of distinct labels by at least $\delta$. Secondly, take an optimal vertex coloring $g$ of graph $G^{p}$ using colors that are integers in the interval $[0, B]$, where $B=\Delta^{p}$ (see proof of Lemma 2.2). Then increase the labels $f^{\prime \prime}(v)$ again, this time by $2 g(v) \varepsilon$.

The labelling obtained after the two augmentations is what we call $f^{\prime}$, and it depends on both $\varepsilon$ and $\delta$. Consider any pair of vertices $v, w \in V$ which are at some distance $i \leq p$ in $G$. If $f^{\prime \prime}(v)=f^{\prime \prime}(w)$ (which can happen only if $k_{i}=0$ ), only the second augmentation changes their difference, and we get that

$$
\left|f^{\prime}(v)-f^{\prime}(w)\right|=|2(g(v)-g(w)) \varepsilon| \geq 2 \varepsilon=\left|f^{\prime \prime}(v)-f^{\prime \prime}(w)\right|+2 \varepsilon,
$$

which is what we claimed. On the other hand, suppose that $f^{\prime \prime}(v) \neq f^{\prime \prime}(w)$, say $f^{\prime \prime}(v)<$ $f^{\prime \prime}(w)$. The first operation must moves their labels at least $\delta$ farther apart, while the second operation may move them closer together, but by at most $2 B \varepsilon$. Let us then specify that $\delta=(2 B+2) \varepsilon$, so that in $f^{\prime}$ the separation between labels for such $v, w$ increases by at least $\delta-2 B \varepsilon=2 \varepsilon$ over $f^{\prime \prime}$. We have that $f^{\prime} \in L\left(\mathbf{k}^{\prime}\right)$.

Now we compare the span of $f^{\prime}$ with that of $f^{\prime \prime}$. The smallest label in $f^{\prime}$ is at least 0 , while the largest label may increase over that in $f^{\prime \prime}$ due to the two operations, by at most $A \delta=A(2 B+2) \varepsilon$ from the first operation, and by at most another $2 B \varepsilon$ from the second operation. Thus, $\operatorname{sp}\left(f^{\prime}\right)-\operatorname{sp}\left(f^{\prime \prime}\right)$ is at most a constant times $\varepsilon$, the constant depending only on $\mathbf{k}$ and $\Delta$, and it goes to 0 with $\varepsilon$.

Next we consider the piecewise linearity of $\lambda(G ; \mathbf{k})$. We say that a function $f$ defined on domain $A \subseteq T^{p}$ is PL on $A$ if it is piecewise linear on $A$ with only finitely many pieces. More specifically, we mean that $A$ can be split by finitely many hyperplanes, such that on each of the closed (polyhedral) regions, $f$ is linear. Further, $f$ is continuous, that is, the linear formulas for two adjacent regions agree on the boundary between them.

We begin with the piecewise linearity for finite graphs, and then consider infinite graphs with bounded degrees.

Theorem 5.2 Let $G=(V, E)$ be a finite graph. Let $p \in \mathbb{Z}^{+}$. Then $\lambda(G ; \mathbf{k})$ is $P L$ as a function of $\mathbf{k}$ on $T^{p}$. Specifically, the domain $T^{p}$ can be split by finitely many hyperplanes 
through the origin into closed convex polyhedral cones, such that $\lambda(G ; \mathbf{k})$ is given by a linear function of the $k_{i}$ 's on each cone.

Proof. Let $G=(V, E)$ be a finite graph on $n$ vertices. Let us partition $T^{p}$ into polyhedral cones by taking all hyperplanes with equations of the form

$$
\sum_{i=1}^{p} b_{i} k_{i}=0, \text { where } \sum_{i}\left|b_{i}\right|<2 n .
$$

By the $D$-set Theorem, for any point $\mathbf{k} \in T^{p}, \lambda(G ; \mathbf{k})$ is the minimum, over feasible labellings $f: V \rightarrow D(\mathbf{k})$, of the maximum label $f(v), v \in V$. Further, it suffices to consider such $f$ in which for all vertices $v, f(v)$ has the form $\sum_{i=1}^{p} a_{i}(v) k_{i}$, where the nonnegative integer coefficients $a_{i}(v)$ satisfy $\sum_{i=1}^{p} a_{i}(v)<n$. (Note that for every $\mathbf{k} \in T^{p}$, there is some feasible labelling, hence some feasible labelling of this form.)

Now we turn things around and fix a labelling $f$ of this form and consider the feasible region for $f$, meaning the set of values $\mathbf{k} \in T^{p}$ for which $f \in L(G ; \mathbf{k})$. We claim that it is a union of convex cones with vertex at the origin.

To see this, note that $\mathbf{k} \in T^{p}$ is feasible for such $f$ whenever it is feasible for each pair of vertices $u, v$ at distance between 1 and $p$. If $u$ and $v$ are at distance $d$, say, this means that $f(u)-f(v)$ is either $\geq k_{d}$ or $\leq-k_{d}$. For the pair $u, v$ the two constraints are bounded by the hyperplanes through the origin with equations $\sum_{i}\left(a_{i}(u)-a_{i}(v)\right) k_{i}=k_{d}$ or $=-k_{d}$, which both have the stated form. Then the feasible region for $f$ is the intersection, over such pairs $u, v$, of these sets in $T^{p}$, each a union (possibly empty) of at most two closed half-spaces. So it is a union of polyhedral cones of the form stated in the theorem.

Within the feasible region of $f$, the maximum label $\max _{v \in V} \sum_{i=1}^{p} a_{i}(v) k_{i}$ depends on k. Chopping the feasible region by all possible comparisons between values of $f$, we get that the feasible region is refined into a union of closed polyhedral cones, bounded by the hyperplanes above plus the additional hyperplanes $f(u)=f(v)$, over distinct $u, v \in V$, that is, $\sum_{i}\left(a_{i}(u)-a_{i}(v)\right) k_{i}=0$, which also has the stated form.

By taking all possible hyperplanes of the stated form $T^{p}$ is divided into polyhedral cones through the origin such that in each such cone (cell) $K$ some nonempty collection of our labellings $f$ is feasible on all of $K$, and for each such feasible $f$, the maximum label $f(v)$ is achieved at a single vertex $v$ (by a single linear formula in $\mathbf{k}$ ). Similarly, the minimum of these maximum labels, over all feasible $f$ on $K$, will be given by a single linear formula (some label $f(v)$ ) throughout $K$.

To summarize, cutting $T^{p}$ by all of the finitely many hyperplanes described above divides it into a finite number of convex polyhedral cones such that $\lambda(G ; \mathbf{k})$ is given by a linear formula of $\mathbf{k}$ in each (closed) cone, and we see $\lambda(G ; \mathbf{k})$ is PL.

We remark that since the formulas for adjacent cells $K$ and $K^{\prime}$ agree on their boundaries, the continuity of $\lambda(G ; \mathbf{k})$ follows for finite graphs $G$. The strength of Theorem 5.1 is evidently that continuity holds as well for infinite graphs $G \in \mathcal{G}_{\Delta}$.

Now consider infinite graphs with bounded maximum degree, say $G \in \mathcal{G}_{\Delta}$. The same arguments above extend, but now the number of hyperplanes cutting through the 
origin is infinite, so the convex cones in the feasible region are not necessarily polyhedral. Nonetheless, we conjecture that Theorem 5.2. can be extended to such infinite graphs:

Let $G=(V, E)$ be a graph, possibly infinite, of bounded maximum degree. Let $p \in \mathbb{Z}^{+}$. Then $\lambda(G ; \mathbf{k})$ is continuous and nondecreasing as a function of $\mathbf{k}$ on $T^{p}$.

Conjecture 5.3 (PL Conjecture) Let $G=(V, E)$ be a graph, possibly infinite, of bounded maximum degree. Let $p \in \mathbb{Z}^{+}$. Then $\lambda(G ; \mathbf{k})$ is $P L$ as a function of $\mathbf{k}$ on $T^{p}$. Specifically, the domain $T^{p}$ can be split by finitely many hyperplanes through the origin into closed convex polyhedral cones, such that $\lambda(G ; \mathbf{k})$ is given by a linear function of the $k_{i}$ 's on each cone.

Despite considerable effort we have not yet succeeded in proving this conjecture. We can give weaker, though still quite strong, results in support of it. One strategy is to restrict the domain by staying away from the coordinate planes (avoiding very small values of the separations $k_{i}$ ): For a number $\varepsilon>0$, let us consider the region $T^{p}(\varepsilon)$ of all points $\mathbf{k}$ with all $k_{i} \geq \varepsilon\left(\sum_{i} k_{i}\right)$. Consider any point $\mathbf{k} \in T^{p}(\varepsilon)$. By Lemma 2.2, $\lambda(G ; \mathbf{k}) \leq \Delta^{p} \sum_{i} k_{i}$. By the $D$-set Theorem there is an optimal labelling in which each label has the form $\sum a_{i} k_{i}$, so that for all $i, a_{i} k_{i} \leq \Delta^{p} \sum_{i} k_{i}$, from which our assumption on $\mathbf{k}$ implies each coefficient is at most a constant, $\Delta^{p} / \varepsilon$. We can then proceed as for Theorem 5.2 and derive the PL property:

Theorem 5.4 Let $G=(V, E)$ be a graph, possibly infinite, of bounded maximum degree. Let $p \in \mathbb{Z}^{+}$. Then for any $\varepsilon>0$ the function $h(\mathbf{k})=\lambda(G ; \mathbf{k})$ is $P L$ on $T^{p}(\varepsilon)$.

Our other supporting result for the PL Conjecture 5.3 is to prove it for conditions out to distance two, that is, for $p=2$. This explains why we obtained PL graphs for $\lambda(G ; k, 1)$ for the graphs we considered. It depends on a special sort of argument that we have been unable to extend to larger $p$. We can derive the PL Theorem for $p=2$ (Theorem 5.6 below) by a different argument in Section 8. We first require some simple bounds on $\lambda\left(G ; k_{1}, k_{2}\right)$ depending on the chromatic number. Note that the upper bound here may be either better or worse than in Lemma 2.2, depending on $k_{1}, k_{2}$.

Lemma 5.5 Let $G=(V, E)$ be a graph, possibly infinite, of maximum degree at most $\Delta>0$. Then

$$
(\chi-1) k_{1} \leq \lambda\left(G ; k_{1}, k_{2}\right) \leq(\chi-1) k_{1}+\chi \Delta^{2} k_{2},
$$

where $\chi$ is the chromatic number of $G$. Also,

$$
\left(\chi\left(G^{2}-G\right)-1\right) k_{2} \leq \lambda\left(G ; k_{1}, k_{2}\right) \leq \chi\left(G^{2}-G\right) \Delta k_{1}+\left(\chi\left(G^{2}-G\right)-1\right) k_{2} .
$$

Proof. We prove the first display; the proof of the second is similar. The lower bound follows easily from

$$
\lambda\left(G ; k_{1}, k_{2}\right) \geq \lambda\left(G ; k_{1}, 0\right)=\lambda\left(G ; k_{1}\right)=k_{1} \lambda(G ; 1)=k_{1}(\chi-1) .
$$


For the upper bound, let us employ two labellings of $G$. First, take any optimal coloring $f_{1}$ of $G$, where the colors are integer labels in $[0, \chi-1]$. (Note that if we instead use a greedy first-fit coloring of $G$, we might not get an optimal coloring; The labels would be in $[0, \Delta]$.) Second, take labelling $f_{2}$ to be a greedy $L(0,1)$-labelling, as in the proof of Lemma 2.2, so that the labels in $f_{2}$ are integers in the interval $\left[0, \Delta^{2}\right]$. Then we define the labelling

$$
f=\left(k_{1}+\Delta^{2} k_{2}\right) f_{1}+k_{2} f_{2} .
$$

By design, $f \in L\left(k_{1}, k_{2}\right)$, and its span is at most

$$
\left(k_{1}+\Delta^{2} k_{2}\right)(\chi-1)+k_{2} \Delta^{2}=(\chi-1) k_{1}+\chi \Delta^{2} k_{2} .
$$

Theorem 5.6 Let $G=(V, E)$ be a graph, possibly infinite, of bounded maximum degree. Then $\lambda\left(G ; k_{1}, k_{2}\right)$ is $P L$ as a function of $\left(k_{1}, k_{2}\right)$ on $T^{2}$. Specifically, the domain $T^{2}$ can be partitioned by finitely many lines through the origin into closed convex polyhedral cones, such that $\lambda\left(G ; k_{1}, k_{2}\right)$ is given by a linear function of the $k_{i}$ 's on each cone.

Proof. Let $G \in \mathcal{G}_{\Delta}$. By the Scaling Property, $\lambda\left(G ; k_{1}, k_{2}\right)=k_{2} \lambda(G ; k, 1)$ for $k_{2}>0$ by setting $k=k_{1} / k_{2}$, so that it suffices to show that $\lambda(G ; k, 1)$, denote this by $g(k)$, is piecewise linear as a function of $k$. The proof of Theorem 5.2 gives us the piecewise linearity we want, except there may be infinitely many linear pieces when $G$ is infinite. It is enough to prove that $g(k)$ is eventually linear for sufficiently large $k$ and also for sufficiently small $k>0$ : Theorem 5.4 guarantees that it is piecewise linear with integer coefficients, with only finitely many pieces, between the linear pieces at the ends.

Let us deal with the case of large $k$. Consider some $k_{o}>\chi \Delta^{2}$. As in the proof of Theorem 5.2, $g\left(k_{o}\right)$ is on a linear segment of the graph of $g$ with a formula of the form $\alpha k+\beta$, where $\alpha, \beta \in \mathbb{N}$. Moreover, the upper bound in Lemma 5.5 gives us that

$$
\alpha k_{o}+\beta \leq(\chi-1) k_{o}+\chi \Delta^{2},
$$

which forces

$$
(\alpha-(\chi-1)) k_{o} \leq \chi \Delta^{2} .
$$

But since $k_{o}>\chi \Delta^{2}$, it must be that $\alpha \leq \chi-1$, as $\alpha$ is integral.

There must be a largest integer coefficient $\alpha$ over the values $k>\chi \Delta^{2}$, say it is $\alpha_{o}$ at $k_{o}$, so that $g\left(k_{o}\right)=\alpha_{o} k_{o}+\beta_{o}$. None of the linear formulas for $k$ larger than $k_{o}$ can be of the form $\alpha_{o} k+\beta$ with $\beta>\beta_{o}$, because there is no way to get above the linear function $\alpha_{o} k+\beta$ without some piece having a slope $\alpha>\alpha_{o}$, contradicting the maximality of $\alpha_{o}$, using the fact that $g(k)$ is continuous. Hence, for all $k \geq k_{o}, g(k) \leq \alpha_{o} k+\beta_{o}$. Then by the lower bound of Lemma 5.5, $(\chi-1) k \leq \alpha_{o} k+\beta_{o}$ for all large $k$. It follows that $\alpha_{o}=\chi-1$.

Linear pieces of the graph of $g(k)$ for $k>k_{o}$ with slope $\alpha_{o}$ must have decreasing values of $\beta$, so that each subsequent linear piece with formula $\alpha_{o} k+\beta$ will have a lower value of $\beta \in \mathbb{N}$. There then must be a last linear piece with $\alpha=\alpha_{o}$. Then this piece never ends, 
because if it did, then after that the slopes would all be at most $\chi-2$, and eventually the graph of $g$ would drop by the lower bound of Lemma 5.5.

It remains to go the other way and show that, for sufficiently small $k, g(k)$ is linear. This is equivalent to showing that $\lambda(G ; 1, k)$ is eventually linear as $k$ grows. The same method used before now works, except the roles of $k_{1}$ and $k_{2}$ are reversed. We conclude that $g(k)$ is eventually linear as $k \rightarrow 0$, with a formula of the form $\alpha k+\chi\left(G^{2}-G\right)$, and it is piecewise linear overall with only finitely many pieces.

\section{Bounds on the Coefficients}

We made a special effort to determine whether the piecewise linearity theorem (Theorem 5.2) holds more generally than for finite graphs: Does it hold for infinite graphs of bounded degree, that is, for the class $\mathcal{G}_{\Delta}$ ? We verified our PL Conjecture 5.3 in cases where we could bound the coefficients $a_{i}$ independent of $\mathbf{k}$. The full PL Conjecture would follow (by the arguments used to prove Theorem 5.2) if one can prove this strengthening of the D-set Theorem:

Conjecture 6.1 (Coefficient Bound Conjecture) Let $G=(V, E)$ be a graph, possibly infinite, of bounded maximum degree. Let $p \in \mathbb{Z}^{+}$. Then there exists a constant $c_{1}=c_{1}(G, p)$ such that for all $\mathbf{k} \in T^{p}$, there is an optimal labelling $f \in L(\mathbf{k})$ with all labels $f(v) \in D(\mathbf{k})$ in which the smallest label is 0 , the largest label is $\lambda(G ; \mathbf{k})$, and each of the labels $f(v)$ and $\lambda(G ; \mathbf{k})$ can be expressed in the form $\sum_{i} a_{i} k_{i}$, where the nonnegative integer coefficients $a_{i}$ are at most $c_{1}$.

We cannot see how to derive the conjecture above from the PL Conjecture. We would need to know more, such as the domain can be split into finitely many regions such that in each region, there is a single labelling of $G$ that is optimal. Of course then there would be a collection of just finitely many labellings $f_{j}$ such that for any $\mathbf{k}$ some labelling in the collection is optimal (and feasible, of course) for $\mathbf{k}$.

We suspect that coefficient bounds can be given that work for all graphs with given maximum degree. Specifically, we propose this strengthening of the Coefficient Bound Conjecture:

Conjecture 6.2 (Delta Bound Conjecture) Let $\Delta, p \in \mathbb{Z}^{+}$. Then there exists a constant $c_{2}=c_{2}(\Delta, p)$ such that for all $\mathbf{k} \in T^{p}$ and all graphs $G=(V, E)$, possibly infinite, of maximum degree at most $\Delta$, there is an optimal labelling $f \in L(G ; \mathbf{k})$ with all labels $f(v) \in D(\mathbf{k})$ in which the smallest label is 0 , the largest label is $\lambda(G ; \mathbf{k})$, and each of the labels $f(v)$ and $\lambda(G ; \mathbf{k})$ can be expressed in the form $\sum_{i} a_{i} k_{i}$, where the nonnegative integer coefficients $a_{i}$ are at most $c_{2}$.

We have not even established this Delta Bound Conjecture yet for general finite graphs $G$, since the bound on the coefficient sums $\sum_{i} a_{i}$ in the $D$-set Theorem, $n-1$, is not restricted by $\Delta$. It does hold trivially for $p=1$ (with coefficient bound $a_{1}=\chi(G)-1 \leq \Delta$. 
It is not clear to us how the proof of the PL Conjecture for $p=2$ (Theorem 5.6) can be used to obtain coefficient bounds to verify the Delta Bound Conjecture for $p>2$. However, we can present another approach, which then gives a different proof of Theorem 5.6, one that may be useful in trying to prove the Delta Bound Conjecture (and, hence, the PL Conjecture) for general $p$.

Theorem 6.3 Let $\Delta \in \mathbb{Z}^{+}$. There exists a constant $c_{3}=c_{3}(\Delta)$ such that for all $\left(k_{1}, k_{2}\right) \in$ $T^{2}$ and all graphs $G=(V, E)$, possibly infinite, of maximum degree at most $\Delta$, there is an optimal labelling $f \in L\left(G ; k_{1}, k_{2}\right)$ with all labels $f(v) \in D\left(k_{1}, k_{2}\right)$ in which the smallest label is 0 , the largest label is $\lambda\left(k_{1}, k_{2}\right)$, and each of the labels $f(v)$ and $\lambda\left(k_{1}, k_{2}\right)$ can be expressed in the form $\sum_{i} a_{i} k_{i}$, where the nonnegative integer coefficients $a_{i}$ are at most $c_{3}$.

Proof. By the Scaling Property, it suffices to prove for given $\Delta$ the existence of $c_{3}$ that works for $(k, 1)$ for all $k \geq 0$. Let $G=(V, E) \in \mathcal{G}_{\Delta}$. Let $f$ be an optimal labelling of $G$ in $L(k, 1)$ as in the $D$-set Theorem.

Case 1. Assume $k$ is very small, say $0<k \leq 1 /\left(2 \Delta^{3}\right)$.

By Lemma 2.2, $f$ has span at most $\Delta^{2}$. Thus, all labels used in $f$ have the form $a k+b$, with nonnegative integer coefficients $a, b$, such that $b \leq \Delta^{2}$. The trouble comes in trying to bound $a$, independent of $k$, no matter how small it gets. What we do is push down the labels $f(v)$ in a greedy way to produce a labelling $f^{\prime} \in L(k, 1)$ such that all labels belong to a set $S \subseteq D(k, 1)$ in which the coefficients $a$ are also bounded in terms of $\Delta$. Since $f^{\prime}$ also has smallest label 0 , and $f^{\prime}(v) \leq f(v)$ for all vertices $v, f^{\prime}$ is an optimal labelling, one that satisfies the required conditions with $c_{3}=\Delta^{3}+\Delta^{2}$.

We define the set

$$
S=\left\{a k+b: a, b \in \mathbb{Z}, 0 \leq b \leq \Delta^{2}, 0 \leq a \leq(b+1) \Delta\right\} .
$$

It is important to note that, by our assumption that $k$ is small means there is a gap between elements in $S$ of the form $a k+b$ and $b+1$. Let the set of labels used by $f$ be given by $\left\{0=l_{0}<l_{1}<l_{2}<\cdots<l_{r}\right\}$, where $r=r(k)$ is finite, since it is contained in the finite set $D(k, 1) \cap\left[0, \Delta^{2}\right]$. For vertices $v$ with label $l_{0}=0$, we set $f^{\prime}(v)=f(v)$. We next take care of vertices in $f^{-1}\left(l_{1}\right)$, then those in $f^{-1}\left(l_{2}\right)$, and so on, through $f^{-1}\left(l_{r}\right)$. Let us suppose we are dealing with vertices $v$ with label $l_{i}$, having already pushed down labels for vertices $w$ with $f(w)<l_{i}$. Although $f^{-1}\left(l_{i}\right)$ can be infinite, no two of its vertices are within distance two, so they can all be pushed simultaneously without any concern about interference. What we do have to ensure is that when we push down $f(v), f^{\prime}(v)$ is not too close to any $f^{\prime}(w)$ for some $w$ already pushed down. We define $f^{\prime}(v)$ to be the largest element of $S \cap\left[0, l_{i}\right]$ that is at least $k$ (resp., 1 ) away from $f^{\prime}(w)$ for every $w$ at distance one (resp., two) from $v$.

With this definition, $f^{\prime}$ has all of the required properties. What needs to be proved is that there is, indeed, some element of $S \cap\left[0, l_{i}\right]$ that is far enough away from the labels $f^{\prime}(w)$ already defined. Put $B=\left\lfloor l_{i}\right\rfloor$ and $R=l_{i}-B$, so that $0 \leq R<1$.

Case 1a. Suppose that $R \leq(B+1) \Delta k$. Put $A=\lfloor R / k\rfloor$. Then $f^{\prime}(v)$ will be $A k+B$. For we have $A k+B \in S, 0 \leq l_{i}-(A k+B)<k$, and $A k+B$ is far enough from values $f^{\prime}(w)$ already 
defined for nearby vertices $w$ : For vertices $w$ at distance one (resp., two) from $v$ that were already pushed, $f(w) \leq f(v)-k=R-k+B$ (resp., $f(w) \leq f(v)-1=R+(B-1)$ ), so that $f^{\prime}(w) \leq(A-1) k+B=f^{\prime}(v)-k$ (resp., $\left.f^{\prime}(w) \leq A k+(B-1)=f^{\prime}(v)-1\right)$.

Case 1b. Suppose that $(B+1) \Delta k<R<1$. Then $f^{\prime}(v)$ will be the largest of the $\Delta+1$ labels $a k+B, B \Delta \leq a \leq(B+1) \Delta$, that is not used as $f^{\prime}(w)$ for any vertices $w$ adjacent to $v$. Then $f^{\prime}(v)$ is at least $k$ away from labels $f^{\prime}(w)$ for $w$ adjacent to $v$. If $w$ at distance two from $v$ was already pushed, then $f(w) \leq R+(B-1)$ means that $f^{\prime}(w) \leq B \Delta k+(B-1)=(B \Delta k+B)-1 \leq f^{\prime}(v)-1$.

Either way, $f^{\prime}(v)$ exists as required.

Case 2. Assume $k$ is very large, say $k \geq 2 \Delta^{4}$.

The argument proceeds as in Case 1, though now we have to use the fact that for any vertex $v$, the number of vertices at distance two is at most $\Delta^{2}$. This time we define our set

$$
S=\left\{a k+b: a, b \in \mathbb{Z}, 0 \leq a \leq \Delta, 0 \leq b \leq(a+1) \Delta^{2}\right\}
$$

We push down $f$ in a similar way to before to produce an optimal labelling $f^{\prime}$ which has coefficient bound roughly $\Delta^{4}$.

Case 3. Assume $k$ is intermediate, $1 /\left(2 \Delta^{3}\right)<k<2 \Delta^{2}$.

By Lemma $2.2, \lambda(k, 1) \leq \Delta^{2} \max \{k, 1\}$, which leads to an upper bound of $2 \Delta^{5}$ on the coefficients $a, b$ of the labels $a k+b$ of the optimal labelling $f$.

We see that $2 \Delta^{5}$ serves as an upper bound on the coefficients for all $k$.

An important note about the Delta Bound Conjecture is that it is simple to give a bound on the label coefficients (in terms of $\Delta$ and $p$ ) for which there does exist a feasible labelling $f$. For instance, there are the labellings described in connection with Lemma 2.2, in which every label has the form $a k$, where $k=\max _{i}\left\{k_{i}\right\}$, and $a$ is at most $\Delta^{p}$. But such labellings are certainly not optimal in general. Hence, the tough part of proving the Delta Bound Conjecture for general $p$ is to show that for some constant $c_{3}$ there exists an optimal feasible labelling with coefficients bounded by $c_{3}$.

Besides being stronger than the PL Conjecture, the Delta Bound Conjecture is perhaps more natural, and easier to understand.

\section{Degree Bounds}

Just as with chromatic numbers, it is interesting to consider how large the optimal span $\lambda(G ; \mathbf{k})$ can be given the degrees of the vertices. Specifically, what if we bound the maximum degree $\Delta(G)$ ? Algorithms devised to achieve bounds we find are potentially useful, since they may produce reasonably efficient channel assignments.

For a connected finite graph $G$, an easy best-possible bound on the chromatic number is

$$
\chi(G) \leq \Delta(G)+1,
$$

and the well-known Brooks's Theorem implies that this bound is best-possible if and only if $G$ is a clique $K_{n}$ or an odd cycle $C_{2 k+1}$. The $\Delta(G)+1$ bound can be achieved by arbitrarily ordering the vertices $V$, say $\left\{v_{1}, v_{2}, \ldots\right\}$, and doing a greedy first-fit labelling 
of them one-by-one (always choose the lowest permissible color). Indeed, this works even if $G$ is infinite.

For the basic $\lambda$-number, $\lambda(G)=\lambda(G ; 2,1)$, the analogous question was proposed in [16], cf. [29]. Of course, a connected graph $G$ with maximum degree 0 or 1 must be a $K_{1}$ or $K_{2}$, respectively, and have $\lambda$ equal to 0 or 2, respectively. After checking many examples, Griggs and Yeh made the still-unproved

Conjecture 7.1 (Delta Squared Conjecture) If $G$ is a connected graph with maximum degree $\Delta \geq 2$, then $\lambda(G) \leq \Delta^{2}$.

This was stated for finite graphs, but would hold as well for infinite graphs, by applying a compactness argument (the Rado Selection Principle, say). The quick explanation for why the bound is quadratic in $\Delta$, instead of linear as for chromatic number, is that the interference in labels extends to distance two from a given vertex, and the number of vertices within distance two can be as large as $\Delta+\Delta(\Delta-1)=\Delta^{2}$. Of course, this observation does not prove the conjecture, since there is the added restriction that labels for adjacent vertices cannot be consecutive.

The conjecture is tantalizing in part because if it fails, it is not by much. Griggs and Yeh used a simple vertex ordering and greedy first-fit labelling to show that

$$
\lambda(G) \leq \Delta^{2}+2 \Delta
$$

which supports the conjecture down to order $O(\Delta)$. On the other hand, they constructed graphs for infinitely many values $\Delta$, using finite projective planes, for which

$$
\lambda(G) \geq \Delta^{2}-\Delta .
$$

Also in support of the conjecture is that it has been shown by many researchers to hold for many classes of graphs. To mention a few, it is known to hold if $G$ is diameter two [16], [29], and better bounds than $\Delta^{2}$ have been proved for trees [16], chordal graphs [28], and planar graphs [18], [26]. Indeed, no one has found any graphs for which the $\Delta^{2}$ bound is sharp, besides the short list in the original paper [16]:

- Paths and cycles, $P_{n}$ and $C_{n}, n \geq 3(\Delta=2)$

- Petersen graph, $n=10(\Delta=3)$

- Hoffman-Singleton graph, $n=50(\Delta=7)$

- the 57-regular diameter-two graph on $57^{2}+1$ vertices, if it exists.

Chang and Kuo [4] managed to cut the gap in the general upper bound in half, proving that

$$
\lambda(G) \leq \Delta^{2}+\Delta
$$

The bound remained there for nearly ten years, before it was improved by Král' and Škrekovski: 
Theorem 7.2 ([21]) Let $G$ be a graph, possibly infinite, with finite maximum degree $\Delta \geq 2$. Then $\lambda(G) \leq \Delta^{2}+\Delta-1$.

For $\Delta=2, G$ must be a path or cycle, for which the conjecture is already verified. The next case up is $\Delta=3$, where the best known general bound is now 11 [21]. Georges and Mauro checked many such graphs [9]. They not only found no graphs with $\lambda(G)>9$, they found no other connected graphs with $\lambda=9$. In fact, they found no such graphs at all with $\lambda=8$, so they suspect (pers. comm.) that $\lambda(G) \leq 7$ if $G$ is connected, has maximum degree 3 , and is not the Peterson graph.

Now we consider $\Delta$-bounds on $\lambda(G ; \mathbf{k})$ for general separations $\mathbf{k}$. Earlier we gave such a bound, again by ordering the vertices and doing a greedy first-fit labelling, in Lemma 2.2:

$$
\lambda(G ; \mathbf{k}) \leq k \Delta^{p}
$$

where $k$ is the maximum $k_{i}$. However, when some $k_{i}$ 's are smaller than $k$, it is clear that $\lambda(G ; \mathbf{k})$ should be smaller. A more careful argument takes advantage of such variation in the separations.

Theorem 7.3 Let $G$ be a graph, possibly infinite, with finite maximum degree $\Delta \geq 0$. Let $\mathbf{k}=\left(k_{1}, \ldots, k_{p}\right) \geq 0$. Then $\lambda(G ; \mathbf{k}) \leq \sum_{i=1}^{p} 2 k_{i} \Delta(\Delta-1)^{i-1}$.

Proof. As in the proof of Lemma 5.5, it is enough to consider a single component of $G$ in which the vertices are arbitrarily ordered $V=v_{1}, v_{2}, \ldots$. We do a greedy first-fit labelling $f$ of the vertices, using for each vertex $v$ the smallest label in $[0, B]$, where $B$ is the bound in the theorem, that is not too close to any previously assigned labels. To see that there is always such an available label, consider a previously labelled vertex $w$ at distance $i$ from $v, 1 \leq i \leq p$. Then $f(v)$ must avoid the interval $\left(f(w)-k_{i}, f(w)+k_{i}\right)$ in order that $f \in L(\mathbf{k})$. Bounding the number of vertices at distance $i$, and assuming in the worst case that all of these vertices are already labelled and that their intervals are disjoint, we have a union of open intervals of lengths adding up to the bound $B$, so some element of $[0, B]$ is available for $f(v)$.

A variation of the argument above gives a related bound that is sometimes slightly better, depending on the $k_{i}$ 's.

Theorem 7.4 Let $G$ be a graph, possibly infinite, with finite maximum degree $\Delta \geq 0$. Let $\mathbf{k}=\left(k_{1}, \ldots, k_{p}\right) \geq 0$. Then $\lambda(G ; \mathbf{k}) \leq \sum_{i=1}^{p}\left(2\left\lceil k_{i}\right\rceil-1\right) \Delta(\Delta-1)^{i-1}$.

Proof. Do a greedy first-fit labelling as before, but restrict the labels $f(v)$ to integers. For a previously labelled vertex $w$ at distance $i$ from $v, f(v)$ must avoid the integers in the interval $\left(f(w)-k_{i}, f(w)+k_{i}\right)$ in order that $f \in L(\mathbf{k})$. These integers are from $f(w)+1-\left\lceil k_{i}\right\rceil$ to $f(w)-1+\left\lceil k_{i}\right\rceil$, a total of $2\left\lceil k_{i}\right\rceil-1$ integers. The stated bound follows as before. 


\section{Related Results}

The development of our theory of real number labellings was influenced by work on the triangular lattice described in the winning student MCM papers [3], [7], [11], [24], [5] and in the preprint [30]. These papers forced us to consider values $\lambda\left(\Gamma_{\Delta} ; k, 1\right)$ for non-integral values of $k$.

There is a considerable amount of work in the literature on labellings that is related to this project. We must first mention earlier work of Georges and Mauro that we only realized, after working out the concept of real-number labellings, is very much in the spirit of this project. In 1995 [8] they proved a restricted version of the $D$-set Theorem: It is shown that for finite graphs $G$ and for integers $p \geq q \geq 0$, there is an optimal labelling in $L(G ; p, q)$ in which every label and $\lambda(G ; p, q)$ have the form $a p+b q$ where $a, b$ are nonnegative integers. They prove in this restricted setting that for integers $c>0$, $\lambda(G ; c p, c q)=c \lambda(G ; p, q)$, a special case of our Scaling Property 2.1. They determine $\lambda(G ; p, q), p \geq q$ for $G$ being a path, a cycle, or various other graphs. In fact, our path and cycle formulas Theorems 3.2 and 3.3 above can be deduced-for $k \geq 1$-from their formulas for integers $p \geq q$ by using our real number model, the Scaling Property 2.1, Corollary 4.2, and continuity (Theorem 5.1).

Moreover, a later paper of Georges and Mauro [10] introduces what we refer to as labellings in $L(G ; k, 1)$ with rational $k \geq 1$. They prove these labellings are continuous. This paper is also maybe the first to consider infinite graphs $G$. Its main result is to determine $\lambda(G ; p, q)$ for integers $p \geq q \geq 1$ when $G$ is the infinite $\Delta$-regular tree.

Early versions of our results were presented at conferences going back to 2001, and slides from a presentation in 2003 are posted on the web [13].

Georges and Mauro (personal communication) have now extended their earlier results to obtain continuity and piecewise-linearity statements for labellings with conditions at distance two, applicable to infinite graphs of bounded degree. This work appears similar to our Theorem 6.3, though their model is more restricted.

Mohar [25] has investigated a more general model, but restricted to finite graphs, in which there is a minimum separation $k_{v, w}$ for every pair of distinct vertices $v$ and $w$. He actually works with the circular span of a graph, proving that it is continuous and piecewise linear, with only finitely many linear segments, as a function of the separations. He also considers an even more general directed graph model. A variation of his argument in the setting of our labellings may give a proof of the PL Conjecture 5.3 (for finite graphs). While our model is more restricted, in that separations depend only the distance between $v$ and $w$, it may have special properties due to this restriction. We also work more generally with infinite graphs.

More recently, a paper by Leese and Noble [23] considers circular real number labellings with conditions at distance two, and obtains a continuous piecewise linear result in that context. 


\section{Directions for Further Research}

Of course, we are anxious to see the conjectures above settled. As we completed this paper, we learned that a group in Prague (Babilon, Jelínek, Král', Valtr) is also preparing a paper on distance-dependent labellings from a somewhat different perspective [1], motivated in large part by the paper of Leese and Noble mentioned above. (Our main ideas were already presented at the DIMACS workshop [13] in October, 2003.)

In the distance two case $(p=2)$ let us consider how soon the formula becomes linear. What John Georges (personal comm.) has observed in many examples is that $\lambda(G ; k, 1)$ seems to be linear for $k>\Delta$-it settles down quickly. Is this true and can it be proven in general? Also, Theorem 6.3 can be used to bound the number of linear pieces in terms of $\Delta$-but how good a bound can be given?

The authors are planning a future paper that explores the symmetry properties of optimal labellings of the triangular lattice with conditions at distance two.

It would be interesting to expand our model to consider infinite graphs with separations $k_{i}$ at all distances $i$, not just finitely many conditions. Even for the particular examples of the triangular lattice and the square lattice, it would be interesting to characterize infinite $\mathbf{k}$ such that $\lambda$ exists.

For use in many applications, we should return to considering the original problem of labelling transmitters in a planar network, using Euclidean distance, rather than graph distance. Perhaps the results for graphs can be helpful?

In some applications we have been told that many channels must be assigned to each transmitter (e.g., if each cellphone user in a particular cell must have a separate frequency). This can be accomplished by assigning an entire arithmetic progression of labels to each vertex in a graph, with the same distance $d$ used for every progression, such that for nearby vertices $v$ and $w$, every label used for $v$ is sufficiently separated from every label for $w$. Equivalently, each vertex is assigned a label in the interval $[0, d)$, with distance between labels measured on the circle, that is, modulo $d$. The goal is to minimize the circular span $d$. There is a sizable literature on this problem for integer labellings. We have been investigating the extension of this model to allow real-number labellings, and there are analogues of some of the results given in this paper for "linear" labellings. This work will be described in a future paper.

\section{Acknowledgements}

The first author wishes to express gratitude to his old friend, Tom Savage, whose enthusiasm for this subject was an inspiration in developing this project. The first author's visit to Trinity, to speak in a special lecture series sponsored by Savage, brought the author together with Trinity faculty John Georges and David Mauro for a stimulating exchange of ideas. Both authors are thankful for helpful discussions and correspondence with many other colleagues, including Tiziana Calamoneri, Daniel Král', Renu Laskar, Daphne D.-F. Liu, Lincoln Lu, László Székely, and Craig Tovey. We also gladly acknowledge the support of the DIMACS-DIMATIA- Rényi series of workshops on graph colorings. 


\section{References}

[1] R. Babilon, V. Jelínek, D. Král', And P. Valtr, Graph labelings with adjustable weights, preprint.

[2] A. A. Bertossi And M. A. Bonuccelli, Code assignment for hidden terminal interference avoidance in multihop packet radio networks, IEEE/ACM Trans. Networking, 3 (Aug., 1995), pp. 441-449.

[3] R. E. Broadhurst, W. J. Shanahan, and M. D. Steffen, We're sorry, you're outside the coverage area, UMAP Journal 21 (2000), pp. 327-342.

[4] G. J. Chang And D. Kuo, The L $(2,1)$-labeling problem on graphs, SIAM J. Discrete Math. 9 (1996), pp. 309-316.

[5] R. Chu, B. XIU, R. Zong, Utilize the limited frequency resources efficiently, UMAP Journal 21 (2000), 343-356.

[6] M. B. Cozzens And F. S. Roberts, T-colorings of graphs and the channel assignment problem, Congr. Numer. 35 (1982), pp. 191-208.

[7] D. J. Durand, J. M. Kline, K. M. Woods, Groovin' with the big band(width), UMAP Journal 21 (2000), pp. 357-367.

[8] J. P. Georges and D. W. Mauro, Generalized vertex labelings with a condition at distance two, Congr. Numer. 109 (1995), pp. 141-159.

[9] J. P. Georges and D. W. Mauro, On generalized Petersen graphs labeled with a condition at distance two, Discrete Math. 259 (2003), pp. 311-318.

[10] J. P. Georges And D. W. Mauro, Labeling trees with a condition at distance two, Discrete Math. 269 (2003), pp. 127-148.

[11] J. Goodwin, D. Johnston, And A. Marcus, Radio channel assignments, UMAP Journal 21 (2000), pp. 369-378.

[12] J. R. GRIGGS, Judge's commentary: The outstanding channel assignment papers, UMAP Journal 21 (2000), pp. 379-386.

[13] J. R. GRIGGS And X. T. Jin, Real number channel assignments with distance conditions, DIMACS Graph Coloring Workshop lecture (Oct., 2003), posted at http://dimacs.rutgers.edu/Workshops/GraphColor/slides.html .

[14] J. R. GRiggs And X. T. Jin, Real number channel assignments for lattices, preprint (Sept., 2005), posted at http://www.math.sc.edu/ griggs/ .

[15] J. R. GRIGGS AND X. T. Jin, Real number labellings for paths and cycles, preprint (Sept., 2005), posted at http://www.math.sc.edu/ griggs/ . 
[16] J. R. GRIGGS AND R. K.-C. YEH, Labelling graphs with a condition at distance 2, SIAM J. Discrete Math. 5 (1992), pp. 586-595.

[17] W. K. Hale, Frequency assignment: Theory and applications, Proc. IEEE 68 (1980), pp. 1497-1514.

[18] J. van Den Heuvel And S. MCGuinness, Coloring the square of a planar graph, J. Graph Th. 42 (2003), pp. 110-124.

[19] X. T. JIN, Real Number Graph Labeling with Distance Conditions, Ph.D. Dissertation, Math. Dept., Univ. of South Carolina, August, 2005.

[20] X. T. JIN AND R. K. YEH, Graph distance-dependent labeling related to code assignment in computer networks, Naval Res. Logis. 52(2005), pp. 159-164.

[21] D. KrÁL' AND R. Škrekovski, A theorem about the channel assignment problem, SIAM J. Discrete Math. 16(2003), pp. 426-437.

[22] R. A. LEESE, A unified approach to the assignment of radio channels to a regular hexagonal grid, IEEE Trans. Vehicular Tech. 46 (1997), pp. 969-980.

[23] R. A. Leese And S. D. Noble, Cyclic labellings with constraints at two distances, Electron. J. Combin. 11 (2004), \#R16, 16pp.

[24] J. Mintz, A. Newcomer, J. C. Prince, A channel assignment model: The span without a face, UMAP Journal 21 (2000), pp. 311-326.

[25] B. MoHAR, Circular colorings of edge-weighted graphs, preprint.

[26] M. Molloy and M. R. Salavatipour, Frequency channel assignment on planar networks, in Proc. 10th Annual Europ. Sympos. Algorithms (ESA) 2002, pp. 736-747.

[27] F. S. RoBerTs, Working Group Agenda, DIMACS/DIMATIA/Renyi Working Group on Graph Colorings and their Generalizations, (2003), posted at http:// dimacs.rutgers.edu/ Workshops/GraphColor/main.html

[28] D. SAKAI, Labelling chordal graphs: distance two condition, SIAM J. Discrete Math. 7 (1994), pp. 133-140.

[29] R. K. YEH, Labeling graphs with a condition at distance 2, Ph.D. dissertation, University of South Carolina, Columbia, SC, 1990.

[30] D. Zhu AND A. SHI, Optimal channel assignments, preprint (2001). 